\title{
The boundary conditions of the heliosphere: photoionization models constrained by interstellar and in situ data
}

\author{
J. D. Slavin ${ }^{1}$ and P. C. Frisch ${ }^{2}$ \\ 1 Harvard-Smithsonian Center for Astrophysics, 60 Garden St., MS 83, Cambridge, MA 02138, USA \\ e-mail: jslavin@cfa.harvard.edu \\ 2 University of Chicago, Department of Astronomy and Astrophysics, 5640 S. Ellis Ave., Chicago, IL 60637, USA \\ e-mail: frisch@oddjob.uchicago.edu
}

Received 15 June 2007 / Accepted 7 May 2008

\begin{abstract}
Context. The boundary conditions of the heliosphere are set by the ionization, density, and composition of inflowing interstellar matter.

Aims. Our aim is to constrain the properties of the Local Interstellar Cloud (LIC) at the heliosphere, which requires radiative transfer ionization models.

Methods. We modeled the background interstellar radiation field using observed stellar FUV and EUV emission and the diffuse soft $\mathrm{X}$-ray background. We also modeled the emission from the boundary between the LIC and the hot Local Bubble plasma, assuming that the cloud is evaporating because of thermal conduction. We created a grid of models covering a plausible range of LIC and Local Bubble properties, and used the modeled radiation field as input to radiative transfer/thermal equilibrium calculations using the Cloudy code. Data from in situ observations of $\mathrm{He}^{0}$, pickup ions and anomalous cosmic rays in the heliosphere, as well as from absorption line measurements towards $\epsilon$ CMa were used to constrain the input parameters.

Results. A restricted range of assumed LIC H I column densities and Local Bubble plasma temperatures produced models that match all the observational constraints. The relative weakness of the constraints on $N(\mathrm{HI})$ and $T_{\mathrm{h}}$ contrast with the narrow limits predicted for the $\mathrm{H}^{0}$ and electron density in the LIC at the Sun, $n\left(\mathrm{H}^{0}\right)=0.19-0.20 \mathrm{~cm}^{-3}$ and $n_{\mathrm{e}}=0.07 \pm 0.01 \mathrm{~cm}^{-3}$. Derived abundances are mostly typical of low-density gas, with sub-solar $\mathrm{Mg}, \mathrm{Si}$, and $\mathrm{Fe}$, possibly subsolar $\mathrm{O}$ and $\mathrm{N}$, and $\mathrm{S}$ about solar; however, $\mathrm{C}$ is supersolar. Conclusions. The interstellar gas at the Sun is warm, low-density, and partially ionized, with $n(\mathrm{H}) \approx 0.23-0.27 \mathrm{~cm}^{-3}, T \approx 6300 \mathrm{~K}$, $X\left(\mathrm{H}^{+}\right) \sim 0.2$, and $X\left(\mathrm{He}^{+}\right) \sim 0.4$. These results appear to be robust since acceptable models are found for substantially different input radiation fields. Our results favor low values for the reference solar abundances for the LIC composition.
\end{abstract}

Key words. ISM: clouds - ISM: abundances - ultraviolet: ISM - X-rays: diffuse background - solar system: general ISM: cosmic rays

\section{Introduction}

The solar wind evacuates a cavity in the interstellar medium (ISM) known as the heliosphere, from which interstellar ions are excluded. In contrast, neutral interstellar gas flows through the heliosphere until destroyed by charge-transfer with the solar wind and photoionization. These neutrals form the parent population of pickup ions (PUI) and anomalous cosmic rays (ACR) observed inside of the heliosphere. The properties of the surrounding interstellar medium set the boundary conditions of the heliosphere and determine its configuration and evolution. An ionization gradient is expected in the cloud feeding ISM into the heliosphere because of the hardness of the interstellar radiation field and the low opacity of the ISM (Cheng \& Bruhweiler 1990; Vallerga 1996; Slavin \& Frisch 2002, hereafter SF02). Because of this ionization gradient, the densities of partially ionized species in the LIC will differ from the values in the circumheliosphere interstellar medium (CHISM) that forms the boundary conditions of the heliosphere. Hence we have undertaken a series of studies to determine the boundary conditions of the heliosphere based on both astronomical and heliospheric data. In turn our results provide tighter constraints on the heliosphere models used to calculate the filtration factors for neutrals that then permit comparisons between ISM inside and outside of the heliosphere.

The distribution and velocity of interstellar $\mathrm{H}^{0}$ inside of the heliosphere were first determined over 30 years ago from the florescence of solar Ly $\alpha$ radiation from these atoms (Thomas \& Krassa 1971; Bertaux \& Blamont 1971; Adams \& Frisch 1977). Similar observations of solar $584 \AA$ florescence from interstellar $\mathrm{He}^{0}$ showed that $\mathrm{H} / \mathrm{He} \sim 6$ for interstellar gas inside of the heliosphere, in contrast to the cosmic value $\mathrm{H} / \mathrm{He}=10$ (Ajello 1978; Weller \& Meier 1981). More recent measurements of $n\left(\mathrm{H}^{0}\right)$ compared to $n\left(\mathrm{He}^{0}\right)$ inside of the heliosphere find a similar ratio of $\mathrm{H} / \mathrm{He} \sim 6-7$ (Richardson et al. 2004; Gloeckler \& Geiss 2004; Witte 2004; Möbius et al. 2004). This difference can be attributed to two effects: the loss of 40-60\% of interstellar $\mathrm{H}^{0}$ due to charge-transfer with protons in the heliosheath region, a process denoted "filtration" (Ripken \& Fahr 1983), and the hardness of the interstellar radiation field at the Sun that ionizes more He than $\mathrm{H}$ (Sect. 4).

The Copernicus satellite first showed that the local interstellar cloud (LIC) surrounding the Sun is low density, $\sim 0.1$ atoms $\mathrm{cm}^{-3}$, partially ionized $\left(n\left(\mathrm{H}^{+}\right) \sim n\left(\mathrm{H}^{0}\right)\right.$, York 1974 ; 
McClintock et al. 1975), and warm (temperature $<10^{4} \mathrm{~K}$, e.g. McClintock et al. 1978). Copernicus, FUSE and HST data have shown that the cluster of local interstellar clouds (low density clouds within $\sim 30 \mathrm{pc}$ ), has low column densities, $N(\mathrm{H} \mathrm{I})<10^{18.7}$, and $N$ ionization levels of $>30 \%$ (e.g. Lehner et al. 2003; Wood et al. 2005), indicating partially ionized gas because $\mathrm{H}$ and $\mathrm{N}$ ionization are coupled by charge-transfer. The low column densities of the LIC itself, $N(\mathrm{HI})<10^{18} \mathrm{~cm}^{-2}$, indicate it is partially opaque to $\mathrm{H}$ ionizing radiation but not to $\mathrm{He}$ ionizing radiation. Cloud opacities of unity are reached for $N(\mathrm{H} \mathrm{I}) \sim 10^{17.2} \mathrm{~cm}^{-2}$ for photons close to the ionization threshold of hydrogen $(13.6 \mathrm{eV})$, and $N(\mathrm{H} \mathrm{I}) \sim 10^{17.7} \mathrm{~cm}^{-2}$ for photons at the $\mathrm{He}^{0}$ ionization edge $(24.6 \mathrm{eV})$. The result is that the LIC is partially ionized with a significant ionization gradient between the edge and center. Because of this radiative transfer effects are important and need to be modeled carefully in order to determine the boundary conditions of the heliosphere.

The LIC belongs to a flow of low density ISM embedded in the very low density and apparently hot $\left(T \sim 10^{6} \mathrm{~K}\right)$ Local Bubble (Frisch 1981; Frisch \& York 1986; McCammon et al. 1983; Snowden et al. 1990). The bulk motion of the local clouds through the local standard of rest ${ }^{1}$ corresponds to a velocity of -17.0 to $-19.4 \mathrm{~km} \mathrm{~s}^{-1}$ from the direction $\ell \sim 331^{\circ}, b \sim-5^{\circ}$ (Frisch \& Slavin 2006). This upwind direction is near the center of the Loop I superbubble and the center of the "ring" shadow that has been attributed to the merging of Loop I and the Local Bubble (Frisch \& York 1986; Frisch 2007; Egger \& Aschenbach 1995). Individual cloudlets with distinct velocities are identified in this flow (Lallement et al. 1986; Frisch et al. 2002). The velocity of the cloud feeding gas into the heliosphere has been determined by the velocity of interstellar $\mathrm{He}^{0}$ in the heliosphere measured by the GAS detector on Ulysses, $-26.3 \mathrm{~km} \mathrm{~s}^{-1}$ (Witte 2004).

Curiously, absorption lines at the LIC velocity are not observed in the nearest interstellar gas in the upwind hemisphere, such as towards the closest star $\alpha$ Cen or towards 36 Oph located $\sim 5$ pc beyond the heliosphere nose (Adams \& Frisch 1977; Landsman et al. 1984; Linsky \& Wood 1996; Wood et al. 2000a,b). This lack of an absorption component at the LIC velocity in the closest stars in the upstream direction indicates that the Sun is near the edge of the LIC, so the CHISM may vary over short distances (and hence timescales).

We are able to test for possible past variations by comparing the first interplanetary $\mathrm{H}^{0}$ Ly $\alpha$ glow spectrum obtained by Copernicus in 1975 with Hubble Space Telescope observations of the Ly $\alpha$ spectra obtained during the mid-1990's solar minimum conditions. The observed $\mathrm{H}^{0}$ velocity and intensity has not varied to within uncertainties over the twenty-year period separating these two sets of observations, so that the CHISM velocity field is relatively smooth over spatial scales of $\sim 120$ AU in the downwind direction (Frisch \& Slavin 2005). The 1975 Copernicus data were acquired in the direction corresponding to ecliptic longitudes of $\lambda=264.3^{\circ}, \beta=+15.0^{\circ}$, or $\sim 13.3^{\circ}$ from the most recent upwind direction derived from SOHO H I Ly $\alpha$ data (Quémerais et al. 2006b,a, Q06). The Copernicus look direction was just outside of the "groove" expected in the Ly $\alpha$ glow in the ecliptic during solar minimum. The groove is caused by increased charge-transfer in the solar wind current sheet, which has a small tilt during minimum conditions (Bzowski 2003). The velocity of the Ly $\alpha$ profile observed by Copernicus corresponds to $-24.8 \pm 2.6 \mathrm{~km} \mathrm{~s}^{-1}$, after correction

\footnotetext{
${ }^{1}$ Heliocentric motions are converted to the local standard of rest, LSR, using the Standard solar apex motion.
}

to the SOHO upwind direction. Since Q06 measured $\mathrm{H}^{0}$ upwind velocities during the solar minimum years of 1996 and 1997 of $-25.7 \pm 0.2 \mathrm{~km} \mathrm{~s}^{-1}$ and $-25.3 \pm 0.2 \mathrm{~km} \mathrm{~s}^{-1}$, which is consistent with the Copernicus results, we must arrive at the conclusion that the flow of interstellar $\mathrm{H}^{0}$ into the heliosphere was relatively constant between the years of 1975 and 1997, so that any observations of variations in the interplanetary Ly $\alpha$ glow properties must be due to solar activity properties alone. The thermal broadening of the Copernicus spectrum corresponded to a temperature of $\sim 5400 \mathrm{~K}$, however measurement uncertainties allowed temperatures of up to $20000 \mathrm{~K}$.

In this paper we present new photoionization models of the LIC and show they reproduce both the densities of the ISM at the heliosphere and column densities in the LIC component towards the star $\epsilon \mathrm{CMa}$. We choose this line of sight for two reasons: the comprehensiveness of the available ion column density data including all of the major coolant elements, and the fact that $\epsilon \mathrm{CMa}$ is the strongest stellar source of EUV flux for the LIC, which makes the simple plane parallel radiative transfer model a better approximation than for other lines of sight. In earlier papers, photoionization models of both the "LIC" and "Blue Cloud" components observed towards $\epsilon$ CMa were used to constrain the models (SF02, Frisch \& Slavin 2003). SF02 grouped the properties of the LIC and Blue Cloud, both of which are with $2.7 \mathrm{pc}$ of the Sun because they are also observed towards Sirius ( $\alpha$ CMa), $\sim 12^{\circ}$ from the $\epsilon$ CMa sightline. The LIC velocity and density are sampled by observations of interstellar He I inside of the heliosphere (-26.3 $\mathrm{km} \mathrm{s}^{-1}$, Witte 2004), and Gry \& Jenkins (2001) show that the properties of the LIC and Blue Cloud differ somewhat. The present study therefore focuses on obtaining the best model of heliosphere boundary conditions by using only data on the LIC inside of the heliosphere, and the LIC component towards $\epsilon$ CMa. The present study also benefits from new atomic rates for the critical $\mathrm{Mg}$ II $\rightarrow \mathrm{Mg}$ I dielectronic recombination coefficient, improved cooling rates in the radiative transfer code Cloudy, recent values for the pickup ion densities at the termination shock, and recent values for solar abundances.

The most truly unique quality of the LIC is that we are inside of it and therefore have the ability to sample the cloud directly via in situ observations carried out by a variety of spacecraft within the Solar System. Of all the available measurements of LIC gas flowing into the Solar System, the observations of the density and temperature of neutral He are apparently the most robust. Helium, unlike hydrogen, undergoes little ionization or heating in traversing the heliosheath regions, no deflection due to radiation pressure, and is destroyed by photoionization and electron-impact ionization within $\sim 1$ AU of the Sun, so we expect that the density and temperature of $\mathrm{He}^{0}$ derived from the observations in the Solar System are truly representative of the values in the LIC (Möbius et al. 2004). In this paper we put a special emphasis on matching these He I data by determining model parameters that yield close agreement with the $n\left(\mathrm{He}^{0}\right)$ and $T\left(\mathrm{He}^{0}\right)$ data simultaneously.

\section{Photoionization model constraints and assumptions}

The primary data constraints on our photoionization models are the LIC component column densities towards $\epsilon$ CMa, (Gry \& Jenkins 2001, hereafter GJ01), in situ observations of $\mathrm{He}^{0}$ (Witte 2004; Möbius et al. 2004), pickup ions (PUI, Gloeckler \& Fisk 2007), and anomalous cosmic rays (ACR, Cummings et al. 2002). The astronomical and in situ observational constraints are summarized in Table 1. 
Table 1. Observational constraints.

\begin{tabular}{llc}
\hline \hline $\begin{array}{l}\text { Observed } \\
\text { Quantity }\end{array}$ & $\begin{array}{l}\text { Observed }^{a} \\
\text { Value }\end{array}$ & Notes $^{b}$ \\
\hline$N(\mathrm{C} \mathrm{II})\left(\mathrm{cm}^{-2}\right)$ & $1.4-2.1 \times 10^{14}$ & $1, \mathrm{~S}$ \\
$N(\mathrm{C} \mathrm{II})\left(\mathrm{cm}^{-2}\right)$ & $1.3 \pm 0.2 \times 10^{12}$ & 1 \\
$N(\mathrm{C} \mathrm{IV})\left(\mathrm{cm}^{-2}\right)$ & $1.2 \pm 0.3 \times 10^{12}$ & 1 \\
$N(\mathrm{~N} \mathrm{I})\left(\mathrm{cm}^{-2}\right)$ & $1.70 \pm 0.05 \times 10^{13}$ & 1 \\
$N(\mathrm{O} \mathrm{I})\left(\mathrm{cm}^{-2}\right)$ & $1.4_{-0.2}^{+0.5} \times 10^{14}$ & $1, \mathrm{~S}$ \\
$N(\mathrm{Mg} \mathrm{I})\left(\mathrm{cm}^{-2}\right)$ & $7 \pm 2 \times 10^{9}$ & 1 \\
$N(\mathrm{Mg} \mathrm{II})\left(\mathrm{cm}^{-2}\right)$ & $3.1 \pm 0.1 \times 10^{12}$ & 1 \\
$N(\mathrm{Si} \mathrm{II})\left(\mathrm{cm}^{-2}\right)$ & $4.52 \pm 0.2 \times 10^{12}$ & 1 \\
$N(\mathrm{Si} \mathrm{III})\left(\mathrm{cm}^{-2}\right)$ & $2.3 \pm 0.2 \times 10^{12}$ & 1 \\
$N(\mathrm{~S} \mathrm{II})\left(\mathrm{cm}^{-2}\right)$ & $8.6 \pm 2.1 \times 10^{12}$ & 1 \\
$N(\mathrm{Fe} \mathrm{II})\left(\mathrm{cm}^{-2}\right)$ & $1.35 \pm 0.05 \times 10^{12}$ & 1 \\
$\left.N(\mathrm{H} \mathrm{I}) / N_{(\mathrm{He}}\right)$ & $14 \pm 0.4^{c}$ & 2 \\
$T(\mathrm{~K})$ & $6300 \pm 340$ & 4 \\
$n(\mathrm{He})\left(\mathrm{cm}^{-3}\right)$ & $0.015 \pm 0.003$ & $4, f=1^{d}$ \\
$n\left(\mathrm{~N}^{0}\right)^{e}\left(\mathrm{~cm}^{-3}\right)$ & $5.47 \pm 1.37 \times 10^{-6}$ & $3, f=0.68-0.95^{d}$ \\
$n\left(\mathrm{O}^{0}\right)^{e}\left(\mathrm{~cm}^{-3}\right)$ & $4.82 \pm 0.53 \times 10^{-5}$ & $3, f=0.64-0.99^{d}$ \\
$n\left(\mathrm{Ne}^{0}\right)^{e}\left(\mathrm{~cm}^{-3}\right)$ & $5.82 \pm 1.16 \times 10^{-6}$ & $3, f=0.84-0.95^{d}$ \\
$n\left(\mathrm{Ar}^{0}\right)^{e}\left(\mathrm{~cm}^{-3}\right)$ & $1.63 \pm 0.73 \times 10^{-7}$ & $3, f=0.53-0.95^{d}$ \\
\hline
\end{tabular}

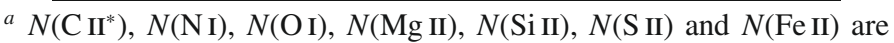
used to constrain the input abundances of the models.

${ }^{b}$ The first number indicates the reference (below), and " $\mathrm{S}$ " indicates that the column density is based on a saturated line. Filtration factors $(f$, Sect. 2.2) are listed for neutrals that have been observed as pickup ions.

${ }^{c}$ The uncertainty given is only that due to uncertainties listed in Dupuis et al. (1995) for the observed H I and He I column densities with the implicit assumption that the ratio is the same on all lines of sight. Given the substantial intrinsic variation in this ratio, however, the quoted uncertainty must be regarded as a lower limit to the true uncertainty.

${ }^{d}$ We assume in this paper that for $\mathrm{He} f_{\mathrm{He}}=1$, and $f$ is not allowed to exceed 1 . Heliosphere models predict the range $f_{\mathrm{He}}=0.92-1$ for $\mathrm{He}$ and $f_{\mathrm{H}}=0.50-0.74$ for $\mathrm{H}$ (see filtration factors in Cummings et al. 2002; Müller \& Zank 2004a; Müller et al. 2008; Izmodenov et al. 2004).

${ }^{e}$ Note that the pickup ion ratios are for values at the termination shock. $\mathrm{N}^{0}, \mathrm{O}^{0}, \mathrm{Ne}^{0}$, and $\mathrm{Ar}^{0}$ densities need to be corrected for filtration in the heliosheath regions (2.2).

References: (1) Gry \& Jenkins (2001). Note that the values shown are for the LIC component towards $\epsilon$ CMa.; (2) Dupuis et al. (1995); (3) Gloeckler \& Fisk (2007); (4) Witte (2004).

\subsection{Astronomical constraints - the LIC towards $\epsilon$ CMa}

The data toward $\epsilon$ CMa of GJ01 show four separate velocity components detected in several different ions including C II, Si III, Si II, and Mg II. One of these velocity components, with a heliocentric velocity of $\sim 17 \mathrm{~km} \mathrm{~s}^{-1}$, is identified as the LIC. Another is identified with a second local cloud, the "Blue Cloud" (BC) at $\sim 10 \mathrm{~km} \mathrm{~s}^{-1}$. In our previous study of LIC ionization (SF02) we noted that since the BC is close to the LIC, and both are detected towards Sirius at $2.7 \mathrm{pc}$ (Lallement et al. 1994; Hébrard et al. 1999), perhaps actually abutting the LIC, we should treat the two as a single cloud for this line of sight. Counter arguments to this line of reasoning include the fact that the BC apparently has different properties than the LIC, though whether it is colder or hotter is unclear (see Hébrard et al. 1999, GJ01). For this reason and because we wish to find the ionization models that best predict interstellar neutral densities inside and outside of the heliosphere, we assume in this paper that the LIC and BC are truly separate clouds and select only the LIC components towards $\epsilon$ CMa to constrain the models.
To justify the identification of the " $17 \mathrm{~km} \mathrm{~s}^{-1}$ " component (or Component 1 ) with the LIC (since $\epsilon$ CMa is some 130 pc away), we note that both Blue Cloud and LIC components are observed on the line of sight towards Sirius which is $12^{\circ}$ from $\epsilon \mathrm{CMa}$. In addition (as pointed out by Gry \& Jenkins 2001) the ratios of ion column densities for the LIC components toward $\epsilon$ CMa and Sirius for several well determined (unsaturated) lines are consistent with each other with ratios $N(\epsilon \mathrm{CMa}) / N($ Sirius $)=1.5 \pm 0.2$. One difference that has been noted between the $\epsilon$ CMa and Sirius lines of sight is the substantial column of Si III detected toward $\epsilon$ $\mathrm{CMa}$, a factor of 10 times larger than the upper limit derived for Sirius (Hébrard et al. 1999). However, as discussed in several papers (Gry \& Jenkins 2001; Slavin 2001; Slavin \& Frisch 2002), $\mathrm{Si}^{++}$cannot have significant abundance in partially ionized warm gas such as the LIC because of the high charge transfer recombination rate with $\mathrm{H}^{0}$. Thus it must be somewhere else along the line-of-sight toward $\epsilon \mathrm{CMa}$, most likely in the boundary between the warm gas and the hot gas of the Local Bubble. The most reasonable explanation for the discrepancy in the Si III columns is then that the cloud boundary is patchy, depending on such things as magnetic field topology or possibly shear flow at any given point in the interface. The evaporative cloud boundaries that we use in this paper cannot account for the Si III column. We intend to explore alternative models for the cloud boundary in future work.

To assess the connection between Component 1 in Gry \& Jenkins (2001) and the circumheliospheric ISM it is necessary to note that the component is actually observed with velocity $16.15 \pm 1.5 \mathrm{~km} \mathrm{~s}^{-1}$ but is labeled as " $17 \mathrm{~km} \mathrm{~s}^{-1}$ " to connect with earlier work that found that value for the projected LIC velocity for the line of sight. The observed velocity vector of $\mathrm{He}^{0}$ flowing into the Solar System (Witte 2004) projected onto the $\epsilon \mathrm{CMa}$ line of sight is $15.08 \pm 0.25 \mathrm{~km} \mathrm{~s}^{-1}$. Thus the velocity of material flowing into the Solar System is consistent with the Component 1 velocity. Moreover both these velocity determinations are consistent with a number of derived velocity vectors for the LIC including new work by Redfield \& Linsky (2008, see also references therein). The $\mathrm{He}^{0}$ projected velocity is not consistent (at the $1-\sigma$ level) with the projected $G$ cloud velocity from Redfield \& Linsky (2008).

In the context of these models, the best astronomical constraints on the neutral ISM component are NI, the saturated O I line, and to some extent $\mathrm{MgI}$ (though the line is weak). The electron density can be deduced from the ratio $\mathrm{Mg} \mathrm{I} / \mathrm{Mg} \mathrm{I}$, which is determined by photoionization and both dielectronic and radiative recombination, and the excitation of the $\mathrm{C}$ II finestructure lines, $\mathrm{C} \mathrm{II} / \mathrm{C}$ II $^{*}$. However, the heavy saturation of the C II $1335 \AA$ line in the ISM limits the accuracy of the determination of $N(\mathrm{C}$ II $)$, so in this paper we have de-emphasized $\mathrm{C} \mathrm{II} / \mathrm{C} \mathrm{II*}$ as an ionization diagnostic. For a discussion of the use of $\mathrm{C} \mathrm{II} / \mathrm{C} \mathrm{II}{ }^{*}$ and other observations in diagnosing the $\mathrm{C}$ abundance and C/S ratio see Slavin \& Frisch (2006). Elements with first ionization potentials (FIP) $<13.6 \mathrm{eV}$ (e.g. $\mathrm{Mg}, \mathrm{Si}, \mathrm{S}$, and $\mathrm{Fe}$ ) are generally almost entirely singly ionized in the LIC and thus the column densities of these ions are close to the total column densities for the elements.

\subsection{In situ Constraints $-\mathrm{He}^{0}$, pickup ions, and anomalous cosmic rays}

Neutral atoms in the LIC penetrate the outer heliosphere regions, and become ionized primarily by charge-transfer with the solar wind ions, photoionization, and electron impact ionization 
(Rucinski et al. 1996). The composition of this neutral population reflects the partially ionized state of the LIC, rather than indicating a pure FIP effect. Thus the observed abundances of neutrals representing high FIP elements, such as $\mathrm{He}, \mathrm{Ne}$, and $\mathrm{Ar}$, as well as $\mathrm{H}, \mathrm{N}$, and $\mathrm{O}$, do not reflect their elemental abundances directly. As a result, these neutrals provide an interesting and unique constraint on the photoionization models. Once ionized, these interstellar wind particles form a population of ions with a distinct velocity distribution that are "picked up" by the solar wind and convected outwards, where they are measured by various spacecraft (Möbius et al. 2004; Gloeckler \& Geiss 2004). These pickup ions (PUI) are accelerated in the heliosheath region and form a population of cosmic rays with an anomalous composition reflective of their origin as interstellar neutrals in a partially ionized gas (Cummings et al. 2002). In situ observations of these byproducts of the ISM interaction with the heliosphere, $\mathrm{H}, \mathrm{He}, \mathrm{N}, \mathrm{O}, \mathrm{Ar}$, and $\mathrm{Ne}$, provide a unique opportunity to constrain theoretical models of an interstellar cloud using the combination of sightline-integrated data, and data from a "single" spatial location, the heliosphere.

We adopt the Ulysses He measurements as the best set of constraints on the ISM inside of the heliosphere. The Ulysses satellite provides direct measurements of interstellar $\mathrm{He}^{0}$ at high ecliptic latitudes throughout the solar cycle (the GAS detector, Witte 2004) and also measurements of the He pickup ion component (the SWICS detector, Gloeckler \& Geiss 2004, 2008). Although interstellar $\mathrm{He}^{0}$ close to the Sun is detected through the resonant scattering of solar $584 \AA$ radiation, geocoronal contamination of the interstellar signal is present so we prefer the Ulysses data (Möbius et al. 2004). We adopt the Ulysses GAS and SWICS results, $n\left(\mathrm{He}^{0}\right)=0.0151 \pm 0.0015 \mathrm{~cm}^{-3}, T\left(\mathrm{He}^{0}\right)=$ $6300 \pm 340 \mathrm{~K}$.

Data on the density of neutral N, O, Ne, and Ar in the surrounding ISM are provided by pickup ion and anomalous cosmic ray data. The densities of interstellar $\mathrm{N}^{0}, \mathrm{O}^{0}, \mathrm{Ne}^{0}$, and $\mathrm{Ar}^{0}$ at the termination shock are listed in Table 1 . These densities must be corrected by the filtration factors, which correspond to the ratios of the densities at the termination shock to those in the LIC. Filtration occurs when neutral interstellar atoms are removed from the inflow by charge-transfer with interstellar protons as the atom crosses the heliosheath regions. Filtration values are listed in Table 1, based on values in Cummings et al. (2002); Müller \& Zank (2004a); Izmodenov et al. (2004). Filtration values larger than 1 are not considered, although some models suggest possible net creation of $\mathrm{O}^{0}$ through charge-transfer between $\mathrm{O}^{+}$ and $\mathrm{H}^{0}$ in the heliosheath regions (Müller \& Zank 2004a). We adopt $f_{\mathrm{He}}=1$ for He. Our models must be compared to the interstellar densities obtained by correcting densities at the termination shock by filtration factors.

\subsection{Interstellar radiation field at the cloud surface}

The spectrum and flux of the cosmic radiation field control the ionization of the very local warm partially ionized medium. The local interstellar radiation field that ionizes the LIC and other nearby cloudlets is determined by the location of the Sun in the interior of a hole in the neutral interstellar gas and dust referred to as the Local Bubble. The clustering tendency of hot $\mathrm{O}$ and $\mathrm{B}$ stars, and attenuation of radiation by interstellar dust and gas, yield the well known spatial variation of the intensity and spectrum of the radiation field. We ignore possible temporal variations of the radiation field (e.g. Parravano et al. 2003), basing our models on observations of the present-day radiation field.

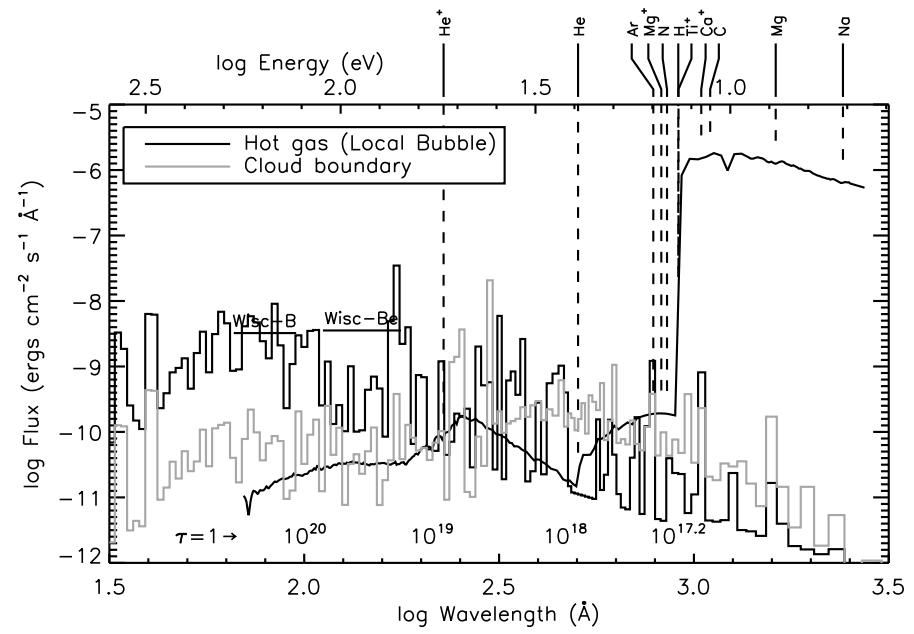

Fig. 1. Modeled interstellar radiation field at the Sun (model 26) as a function of wavelength (bottom $X$-axis) and energy (top $X$-axis). The black histogram is the modeled hot gas (i.e. Local Bubble) spectrum while the gray histogram is the cloud boundary contribution. The other line is the stellar EUV/FUV background. The list of elements at the top of the plot identifies the ionization potentials for neutrals and ions of interest. The energy/wavelength at which an optical depth of 1 is reached for several different H I column densities is shown along the bottom of the plot. Observed flux levels of the soft X-ray diffuse background in the Wisconsin Be and $B$ bands are plotted as lines (Bloch et al. 1986; McCammon et al. 1983).

The interstellar radiation field we use in our models is based on observations of the radiation field at the Sun, supplemented by theoretical calculations of the spectra in the EUV and soft $\mathrm{X}$-rays where lack of sensitivity and/or spectral resolution require the use of models to create a realistic spectrum. The directly observed radiation field includes the far ultraviolet (FUV) field created primarily by B stars, the extreme ultraviolet (EUV) field from two B stars ( $\epsilon$ CMa and $\beta$ CMa) and hot nearby whitedwarf stars, and the diffuse soft X-ray background (SXRB). We show the radiation field at the cloud surface in Fig. 1. Because the interstellar opacity for radiation with $E>13.6 \mathrm{eV}$ is vastly greater than for $E<13.6 \mathrm{eV}$, the EUV part of the radiation field originates in nearby regions with $N(\mathrm{HI}) \lesssim 10^{18} \mathrm{~cm}^{-2}$ while the FUV comes from a much larger volume.

We use the EUV field of Vallerga (1998), which is based on data collected by the Extreme Ultraviolet Explorer (EUVE) satellite. The EUVE spectrometers were sensitive over the wavelength range of 504-730 $\AA$ and showed that the stellar part of the EUV background is dominated by $\epsilon$ CMa and $\beta$ CMa with substantial contributions from nearby hot white dwarfs at shorter wavelengths. Vallerga extrapolated those measurements to the $\mathrm{H}^{0}$ ionization edge at $912 \AA$ using a total interstellar $\mathrm{H}^{0}$ density towards $\epsilon$ CMa of $N(\mathrm{HI})=9 \times 10^{17} \mathrm{~cm}^{-2}$. This value for $N(\mathrm{HI})$ appears somewhat high based on observations of Gry \& Jenkins (2001) which, though dependent on assumptions for gas phase abundances, indicate a value for $N(\mathrm{H} \mathrm{I})$ of $\sim 7 \times 10^{17} \mathrm{~cm}^{-2}$. This uncertainty in the total $N(\mathrm{HI})$ affects only the extrapolated portion of the spectrum in our models since the other portion of the spectrum is derived by de-absorbing the observed spectrum by the value of $N(\mathrm{HI})$ assumed just for the LIC. For the results presented here we have assumed that the total $N(\mathrm{HI})$ towards $\epsilon$ CMa is $7 \times 10^{17} \mathrm{~cm}^{-2}$. Assuming a larger value would increase the flux in the extrapolated region, though our calculations indicate that the overall affect on our results is only at the $\sim 2 \%$ level at most. 
The FUV field is important because it sets the ionization rate of $\mathrm{Mg}^{0}$, which has a first ionization potential of $7.65 \mathrm{eV}$ (1621 $\AA$ ). The radiation field shortwards of $1600 \AA$ is heavily dominated by $\mathrm{O}$ and $\mathrm{B}$ stars in Gould's Belt, particularly those occupying the unattenuated regions of the third and fourth galactic quadrants. A pronounced spatial asymmetry in the $1565 \AA$ radiation field has been observed by the TD- 1 satellite $\mathrm{S} 2 / 68$ telescope survey of the interstellar radiation field, and we use those data and the extrapolation down to $912 \AA$ from the $1564 \AA$ measurements as calculated by Gondhalekar et al. (1980). The asymmetries in the TD-1 $1565 \AA$ radiation field are reproduced by diffuse interstellar radiation field models (Henry 2002).

The diffuse soft X-ray background (SXRB) has been observed over the entire sky at relatively low spatial and spectral resolution by ROSAT (Snowden et al. 1997) and proportional counters flown on sounding rockets by the Wisconsin group (e.g., McCammon et al. 1983). The broadband count rates in the low energy bands, particularly the $B$ and $C$ bands (130-188 eV and 160-284 eV respectively) have been modeled as coming from an optically thin, hot plasma at a temperature of $\sim 10^{6} \mathrm{~K}$ that occupies the low density cavity extending to $\sim 50-200$ pc from the Sun in all directions (Snowden et al. 1990). Refinements to this picture have been required by ROSAT data showing absorption by relatively distant clouds (e.g. MBM12, Snowden et al. 1993). Snowden et al. (1998) propose a picture in which the emission is divided between a Local Bubble component (unabsorbed except for the LIC) and a distant absorbed component, mainly in the Galactic halo. More recently there has been growing evidence that some, possibly large, fraction of the SXRB is generated within the heliosphere from charge exchange between solar wind ions and neutral atoms. We discuss this further in Sect. 5.6.

We model the spectrum measured by the broad-band soft X-ray observations by assuming that the SXR background consists of a local (unabsorbed) component and a distant (halo) component absorbed by an H I column density of $10^{19} \mathrm{~cm}^{-2}$. The emission measure or intrinsic intensity of the local and distant components are assumed to be equal. The spectrum is calculated using the Raymond \& Smith (1977, updated) plasma emission code assuming a hot, optically thin plasma in collisional ionization equilibrium. We explore temperatures for the hot gas of $\log T_{\mathrm{h}}=5.9,6.0$ and 6.1. The total flux, scaled by the emission measure, is fixed so that the $B$ band flux matches the all-sky average from McCammon et al. (1983).

The boundary region between the warm LIC and the adjacent Local Bubble plasma may be another significant source of EUV radiation and we include this flux in our models 1-30. We model this transition region as a conductive interface between the LIC and Local Bubble plasma in the same way as described in Slavin \& Frisch (2002). The cloud is assumed to be steadily evaporating into the surrounding hot gas. The partially ionized gas of the LIC is heated and ionized as it flows into the Local Bubble. The ionization falls out of equilibrium, with low ionization stages persisting into the hot gas. The non-equilibrium ionization is followed and the emission in the boundary is calculated again using the Raymond \& Smith (1977) code. Ions in the outflow are typically excited several times before being ionized and the boundary region radiates strongly in the $13.6-54.4 \mathrm{eV}$ band. The contribution of the interface emission to the total $B$ band flux is taken into account in the calculation of the emission measure for the hot gas of the Local Bubble so that the $B$ band flux still matches the all-sky average. As an aside we point out that our models, for a variety of reasons including the
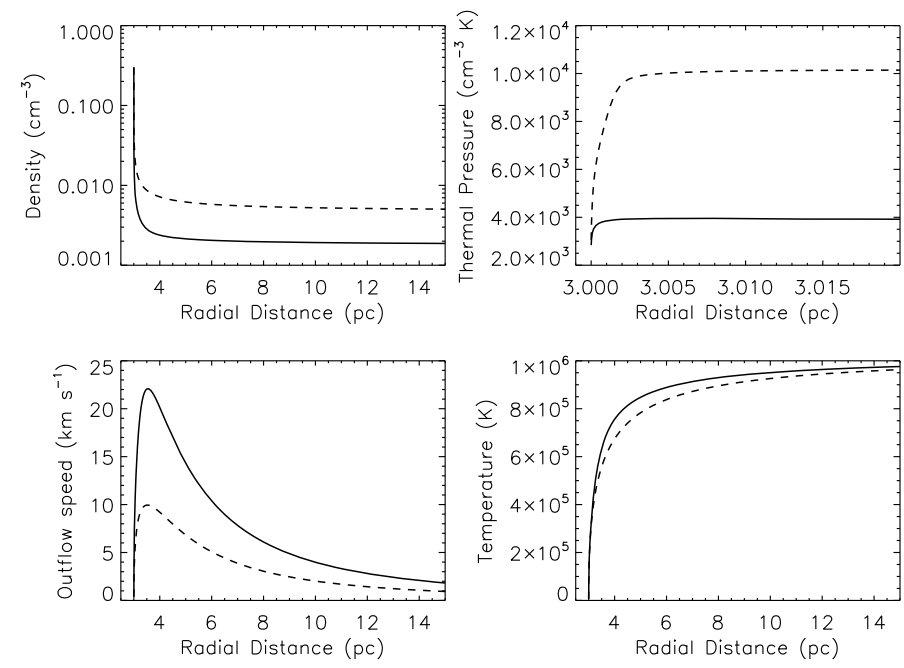

Fig. 2. Profiles of hydrodynamic variables in two different cloud boundary calculations. The solid lines are for model 6 which has $n_{\mathrm{H}}=$ $0.273 \mathrm{~cm}^{-3}, \log T_{\mathrm{h}}=6.0, B_{0}=2 \mu \mathrm{G}$ and $N_{\mathrm{HI}}=4.5 \times 10^{17} \mathrm{~cm}^{-2}$, while the dashed lines are for model 8 , which differs from model 6 only in the strength of the magnetic field, $B_{0}=5 \mu \mathrm{G}$. Note that in the upper right panel, the plot of thermal pressure, the radial scale is much smaller in order to show the variation in thermal pressure. The higher magnetic pressure inside the cloud for model 8 leads to the higher external thermal pressure for that case. The temperature profile differs in the two cases because the degree of heat flux saturation is reduced for the higher thermal pressure of model 8, which in turn leads to a shallower temperature gradient.

O abundance, amount of radiative cooling, reduced conductivity (one half the Spitzer value), and saturation effects, predict only modest column densities of $\mathrm{O}$ VI from the cloud boundary, $\log N(\mathrm{O} \mathrm{VI})\left(\mathrm{cm}^{-2}\right)=12.3-12.8$.

We note that no attempt is made to make this model consistent with the size of the local cavity, which is proposed to contain the hot gas in the standard model for the SXRB (e.g., Snowden et al. 1998). The pressure in the hot gas is not adjusted to fit such models. Rather the total pressure in the hot gas for an evaporating cloud model is dictated by the assumed density, temperature and magnetic field in the cloud. In fact the pressures in our models come out far too low for the standard model to produce the SXRB within the confines of the Local Cavity as deduced, e.g., from Na I observations (Lallement et al. 2003). This in turn means that if the thermal pressure in the Local Bubble turns out to be much lower than was assumed in those models because a substantial fraction of the SXRB comes from solar wind charge exchange emission then the emission from the cloud boundary would be unaffected.

An example of the profiles of the hydrodynamic variables for two different models is shown in Fig. 2. The magnetic field strength in these calculations is assumed to be proportional to the density at every point in the outflow. The treatment here is the same as in Slavin (1989) which contains a more thorough discussion of the issues involved in this sort of calculation. The effect of the field on the conductivity is parametrized in a simple way by a constant reduction factor of 0.5 . The importance of the magnetic field for our calculations lies in the way it affects the thermal pressure in the layer. Since the density drops sharply in the outflow and $|B| \sim n$, any magnetic pressure $\left(\sim B^{2}\right)$ drops even more sharply. The total pressure is roughly constant in the boundary, so the thermal pressure necessarily rises to make up for the decreasing magnetic pressure. Since all our models have 
nearly the same thermal pressure in the cloud, the primary effect of the magnetic field is to help determine the thermal pressure in the interface and thus radiative flux from the boundary. Since the total soft X-ray flux is fixed by requiring a match with the Wisconsin $B$ band all-sky average count rate (McCammon et al. 1983), a larger assumed magnetic field increases only the EUV flux which is not constrained by the $B$-band data. The affect on the cloud of a larger EUV flux is to increase its temperature and ionization.

A secondary effect of the magnetic field can be seen in the temperature profiles in Fig. 2. In evaporating clouds in the ISM, if the temperature gradient is large enough and the thermal pressure is low enough, the conduction becomes "saturated", which means that the heat flux expected from the gas temperature and temperature gradient exceeds the flux that can be carried by the electrons (Cowie \& McKee 1977). Saturation leads to a steepening of the temperature gradient and a relative slowing of the mass loss rate. In the two cases shown in Fig. 2, the lower magnetic field case $\left(B_{0}=2 \mu \mathrm{G}\right)$ is moderately saturated (in terms of Cowie \& McKee's parameter, $\sigma_{0}=3$ ) while the other case $\left(B_{0}=5 \mu \mathrm{G}\right)$, because of the higher magnetic pressure, is less saturated.

\section{Photoionization models}

The photoionization models of the LIC are developed following the same underlying procedure as in SF02, but selecting only the LIC absorption component towards $\epsilon$ CMa for comparison. Improvements include using updated values for in situ ISM observations and using a recent version of the Cloudy radiative transfer/thermal equilibrium code (version 06.02.09a, Ferland et al. 1998). We run Cloudy with the assumption of a planeparallel cloud geometry and format our calculated photoionizing spectrum to be used as input. The selected options include utilizing recent calculations for the dielectronic recombination rates for $\mathrm{Mg}^{+} \rightarrow \mathrm{Mg}^{0}$ (e.g. Altun et al. 2006, and the commands "set dielectronic recombination Badnell" and "set radiative recombination Badnell"), the assumption of constant pressure, and the inclusion of interstellar dust grains at $50 \%$ abundance compared to a standard ISM value. As we discuss below, the only role that dust plays is net heating of the gas since there is far too little column for extinction to be important (expected $E(B-V) \sim 10^{-4.2}$ ). The fraction of the heating provided by dust is $\sim 4 \%$ of the total heating, while dust provides $\sim 2 \%$ of the cooling (mainly by capture of electrons onto grain surfaces). A cosmic ray ionization rate at the default background level of $2.5 \times 10^{17} \mathrm{~s}^{-1}$ (for $\mathrm{H}$ ionization) is included. The LIC is assumed to be in ionization and thermal equilibrium, and Cloudy calculates the detailed transfer of radiation, including absorption and scattering of the radiation incident on the cloud surface, as well as the diffuse continuum and emission lines generated within the cloud.

The procedure for creating a model begins with generating the incident radiation field at the cloud surface (Sect. 2.3). The radiative transfer model is then run, and the output predictions of the model are compared to observations of interstellar absorption lines in the LIC towards $\epsilon \mathrm{CMa}\left(N\left(\mathrm{C} \mathrm{II}^{*}\right), N(\mathrm{~N} \mathrm{I}), N(\mathrm{O} \mathrm{I})\right.$, $N(\mathrm{Mg}$ II $), N(\mathrm{~S}$ II $), N(\mathrm{Si}$ II $), N(\mathrm{Fe}$ II $))$ and in situ observations of $n\left(\mathrm{He}^{0}\right)$ by spacecraft inside of the solar system. The abundances of $\mathrm{C}, \mathrm{N}, \mathrm{O}, \mathrm{Mg}, \mathrm{Si}, \mathrm{S}$ and $\mathrm{Fe}$ are then adjusted to be consistent with the observed column densities. With these new abundances, the Cloudy run is repeated and this process is continued until no adjustment of the abundances is needed. Because the abundances do have an impact on the emission from the cloud boundary, the cloud evaporation model is then re-run with the new abundances

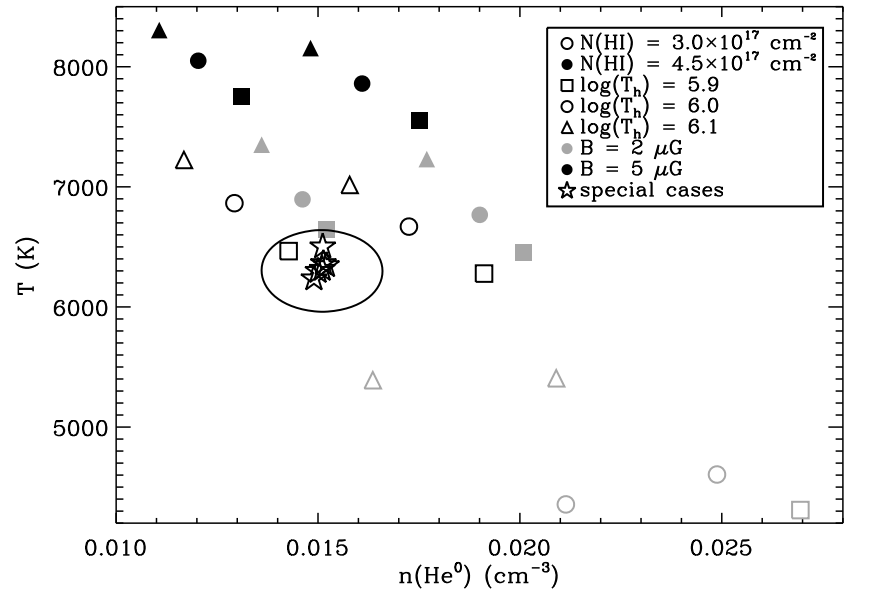

Fig. 3. Model results for the $\mathrm{He}^{0}$ density and temperature in the ISM just outside the heliosphere. The squares, circles, and triangles are for models that are part of the initial grid of 24 models, while the stars are for models 25-30 for which the magnetic field, $B$, and the total $\mathrm{H}$ density, $n_{\mathrm{H}}$, were varied to match the observed $n\left(\mathrm{He}^{0}\right)$ and $T$. For the grid models, the empty symbols are for models with $N(\mathrm{HI})=3 \times$ $10^{17} \mathrm{~cm}^{-2}$, while the filled symbol models have $N(\mathrm{H} \mathrm{I})=4.5 \times 10^{17} \mathrm{~cm}^{-2}$. As the legend shows, the color (black vs. gray) indicates the magnetic field strength and the symbol shape indicates the temperature assumed for the hot gas of the Local Bubble. For identical kinds of points, the one to the left is for a model with $n\left(\mathrm{H}^{0}\right)=0.218 \mathrm{~cm}^{-3}$ and the one to the right has $n\left(H^{0}\right)=0.273 \mathrm{~cm}^{-3}$. The ellipse is the error range around the observed values for $n\left(\mathrm{He}^{0}\right)$ and $T$. Special cases are discussed in Sect. 4.

as well to re-generate the input ionizing spectrum. The iterative process of generating the spectrum and doing the Cloudy photoionization runs generally requires only a couple runs of the cloud evaporation program and a few runs of Cloudy.

\section{Model results}

To investigate the dependence of the results on the input parameters we calculate a grid of 24 models. We explore total $\mathrm{H}$ density, $n_{\mathrm{H}}=0.273,0.218 \mathrm{~cm}^{-3}$; Local Bubble hot gas temperature, $\log T_{\mathrm{h}}(\mathrm{K})=5.9,6.0,6.1$; magnetic field strength, $B_{0}=2,5 \mu \mathrm{G}$; and $\mathrm{HI}$ column density, $N_{\mathrm{HI}}=3 \times 10^{17}, 4.5 \times 10^{17} \mathrm{~cm}^{-2}$. The values for $T$ and $n(\mathrm{HeI})$ at the solar location, the endpoint of the calculations, are shown in Fig. 3 for this set of models. We then explore another six models in which we do the calculations over a grid in $\log T_{\mathrm{h}}$ and $N_{\mathrm{HI}}$ but vary the values of $n_{\mathrm{H}}$ and $B_{0}$ in order to match the observed $T$ and $n(\mathrm{He} \mathrm{I})$. We employed a multiple linear regression to assist in narrowing down the search region for the values of $n_{\mathrm{H}}$ and $B_{0}$ needed to match the observations. Since the dependencies of the results on the parameters really are quite non-linear, this procedure could not work to predict exactly correct values for the required parameters, but was useful for getting close to the correct values. Based on the results for the initial grid of models, we use $\log T_{\mathrm{h}}=5.9,6.0,6.1$ and $N_{\mathrm{HI}}=3 \times 10^{17}, 4 \times 10^{17} \mathrm{~cm}^{-2}$ for this smaller grid, models $25-30$. We chose to use $4 \times 10^{17} \mathrm{~cm}^{-2}$ rather than $4.5 \times 10^{17} \mathrm{~cm}^{-2}$ because the higher column density models, for the most part, produced temperatures that were too high. As can be seen from Fig. 3, all of these models (25-30), plotted as stars, are consistent with the observed $T$ and $n(\mathrm{He} \mathrm{I})$, indicated by the ellipse in the figure. Table 2 gives the input parameters for each model. Model predictions for the $\epsilon$ CMa sightline integrated through the LIC are 
Table 2. Model input parameter values.

\begin{tabular}{|c|c|c|c|c|}
\hline \multirow[b]{2}{*}{ Model No. } & \multicolumn{4}{|c|}{ Input parameter } \\
\hline & $\begin{array}{c}n_{\mathrm{H}} \\
\left(\mathrm{cm}^{-3}\right)\end{array}$ & $\begin{array}{c}\log T_{\mathrm{h}} \\
(\mathrm{K})\end{array}$ & $\begin{array}{c}B_{0} \\
(\mu \mathrm{G})\end{array}$ & $\begin{array}{c}N_{\mathrm{HI}} \\
\left(10^{17} \mathrm{~cm}^{-2}\right)\end{array}$ \\
\hline 1 & 0.273 & 5.9 & 2.0 & 3.0 \\
\hline 2 & 0.273 & 5.9 & 2.0 & 4.5 \\
\hline 3 & 0.273 & 5.9 & 5.0 & 3.0 \\
\hline 4 & 0.273 & 5.9 & 5.0 & 4.5 \\
\hline 5 & 0.273 & 6.0 & 2.0 & 3.0 \\
\hline 6 & 0.273 & 6.0 & 2.0 & 4.5 \\
\hline 7 & 0.273 & 6.0 & 5.0 & 3.0 \\
\hline 8 & 0.273 & 6.0 & 5.0 & 4.5 \\
\hline 9 & 0.273 & 6.1 & 2.0 & 3.0 \\
\hline 10 & 0.273 & 6.1 & 2.0 & 4.5 \\
\hline 11 & 0.273 & 6.1 & 5.0 & 3.0 \\
\hline 12 & 0.273 & 6.1 & 5.0 & 4.5 \\
\hline 13 & 0.218 & 5.9 & 2.0 & 3.0 \\
\hline 14 & 0.218 & 5.9 & 2.0 & 4.5 \\
\hline 15 & 0.218 & 5.9 & 5.0 & 3.0 \\
\hline 16 & 0.218 & 5.9 & 5.0 & 4.5 \\
\hline 17 & 0.218 & 6.0 & 2.0 & 3.0 \\
\hline 18 & 0.218 & 6.0 & 2.0 & 4.5 \\
\hline 19 & 0.218 & 6.0 & 5.0 & 3.0 \\
\hline 20 & 0.218 & 6.0 & 5.0 & 4.5 \\
\hline 21 & 0.218 & 6.1 & 2.0 & 3.0 \\
\hline 22 & 0.218 & 6.1 & 2.0 & 4.5 \\
\hline 23 & 0.218 & 6.1 & 5.0 & 3.0 \\
\hline 24 & 0.218 & 6.1 & 5.0 & 4.5 \\
\hline 25 & 0.226 & 5.9 & 4.7 & 3.0 \\
\hline 26 & 0.213 & 5.9 & 2.5 & 4.0 \\
\hline 27 & 0.226 & 6.0 & 3.8 & 3.0 \\
\hline 28 & 0.216 & 6.0 & 2.1 & 4.0 \\
\hline 29 & 0.232 & 6.1 & 3.4 & 3.0 \\
\hline 30 & 0.223 & 6.1 & 0.05 & 4.0 \\
\hline 42 & 0.218 & 6.1 & - & 4.5 \\
\hline
\end{tabular}

presented in Table 3. Model predictions for the CHISM (i.e. at the solar location) are shown in Table 4.

In SF02 we tested models with no emission from the cloud boundary. This amounts to assuming that the boundary is a sharp transition from the hot gas of the Local Bubble to the warm gas of the LIC. We have again explored such models using the LIC data as constraints in the same way as for the models discussed above with a conductive interface. When there is no evaporative boundary, our models do not depend on the magnetic field in the cloud since in that case the ionizing flux consists only of diffuse emission from the hot gas of the Local Bubble and EUV emission from nearby hot stars and the spectrum is not related to the properties of the cloud. For these models our model grid consists of total $\mathrm{H}$ density, $n_{\mathrm{H}}=0.273,0.218 \mathrm{~cm}^{-3}$, Local Bubble hot gas temperature, $\log T_{\mathrm{h}}(\mathrm{K})=5.9,6.0,6.1$, and $\mathrm{H}$ I column density, $N_{\mathrm{HI}}=3 \times 10^{17}, 4.5 \times 10^{17} \mathrm{~cm}^{-2}$, for a total of twelve models that we label as models $31-42$. Of these models, however, only two resulted in ionizing fluxes sufficient to heat the cloud to a temperature $\sim 6000 \mathrm{~K}$ at the same time as matching the constraints on the ion column densities. These models were the ones with $\log T_{\mathrm{h}}=6.1$ and $N_{\mathrm{HI}}=4.5 \times 10^{17} \mathrm{~cm}^{-2}$ (models 36 and 42). For the other cases, either at the surface or deeper into the cloud, there are insufficient photons to provide the heating to balance the cooling and the cloud temperature drops sharply to $<1000 \mathrm{~K}$. The model with $n_{\mathrm{H}}=0.218$ (model 42$)$ is consistent with the observed value of $n\left(\mathrm{He}^{0}\right)$ and with the column density data as well.

From these 42 models we have selected those that provide acceptable results for the observational constraints, according to prioritized requirements. The first requirement is that the model predict the density and temperature of $\mathrm{He}$ I observed inside of the solar system. Models 14 (marginally), 15 and 25-30 and 42 predict a He density and temperature consistent with the observed values within the reported errors. They also predict the pickup ion $\mathrm{Ne}$ densities, for an assumed $\mathrm{Ne} / \mathrm{H}=123 \mathrm{ppm}$. Models 26 and 28 successfully match the PUI data for $\mathrm{Ne} / \mathrm{H}$ as low as $\sim 100 \mathrm{ppm}$. These models are also required to match the observed $\mathrm{Mg}$ II/Mg I ratio in the LIC towards $\epsilon$ CMa. Models 14, 27, and 29 marginally fit this criterion, while 26, 28, 30, and 42 successfully fit this criterion. Note that the new models now provide acceptable predictions for the $\mathrm{He}^{0}$ temperature at the solar location, which was not the case for the best models in SF02. These models are also consistent with the observed $\mathrm{C} \mathrm{II} / \mathrm{C} \mathrm{II*}$ ratios in the LIC component towards $\epsilon$ CMa though this constraint is weak because of the large uncertainties in $N(\mathrm{C}$ II $)$. Based on these comparisons, and given the uncertainties in both data and models, Models 14, 26-30, and 42 are plausible models, but Models 26 and 28 appear to best match the observational constraints.

Based on the constraints and assumptions presented in the previous section, we select models $14,26-30$ and 42 as the best models for the LIC ionization, with models 26 and 28 favored by the PUI Ne data provided that $\mathrm{Ne} / \mathrm{H}>100 \mathrm{ppm}$. We believe that these seven models then give a realistic range for the uncertainties in the boundary conditions of the heliosphere, providing that the underlying assumptions implicit in the Cloudy code, e.g. photoionization equilibrium, are correct. The predictions of the best models provide excellent matches to the observational constraints. Based on these models, we find the boundary conditions of the heliosphere to be describable as $n\left(\mathrm{H}^{0}\right)=0.19-0.20 \mathrm{~cm}^{-3}, n_{\mathrm{e}}=0.05-0.08 \mathrm{~cm}^{-3}$, and $X(\mathrm{H}) \equiv \mathrm{H}^{+} /\left(\mathrm{H}^{0}+\mathrm{H}^{+}\right)=0.19-0.26, X(\mathrm{He}) \equiv \mathrm{He}^{+} /\left(\mathrm{He}^{0}+\right.$ $\left.\mathrm{He}^{+}\right)=0.36-0.43$. For these models, we find abundances of $\mathrm{O} / \mathrm{H}=295-437 \mathrm{ppm}, \mathrm{C} / \mathrm{H}=589-813 \mathrm{ppm}$, and $\mathrm{N} / \mathrm{H}=$ 40.7-64.6 ppm (Table 7). The total LIC density is $n(\mathrm{H})_{0}=$ $0.213-0.232 \mathrm{~cm}^{-3}$, while the strength of the interstellar magnetic field in the cloud varies between 0 and $3.8 \mu \mathrm{G}$. (Note that the subscript 0 denotes the value at the cloud face. The total (neutral + ionized) $\mathrm{H}$ density varies through the cloud.) The Ne PUI data further favor densities of $n\left(\mathrm{H}^{0}\right) \approx 0.19 \mathrm{~cm}^{-3}$ and $n_{\mathrm{e}}=0.06-0.07 \mathrm{~cm}^{-3}$.

In this analysis we have assumed negligible filtration for $\mathrm{He}$ in the heliosheath regions. Modeling of the He filtration factor however allows values as small as $f_{\mathrm{He}}=0.92$, which yields $n(\mathrm{He} \mathrm{I})=0.0164 \mathrm{~cm}^{-3}$ for the CHISM (Table 1). The predictions of Model 21 agree with this value for $n(\mathrm{He} \mathrm{I})$, as well as with the ratios $\mathrm{Mg}$ II/Mg I and $\mathrm{C} \mathrm{II} / \mathrm{C} \mathrm{II}^{*}$ towards $\epsilon$ CMa and the pickup ion $\mathrm{Ne}$ and $\mathrm{Ar}$ data (Table 3 ). The predicted cloud temperature is low by $\sim 1000 \mathrm{~K}$. The density and ionization for Model 21 are $n\left(\mathrm{H}^{0}\right)=0.21 \mathrm{~cm}^{-3}$ and $n_{\mathrm{e}}=0.06 \mathrm{~cm}^{-3}$. We therefore conclude that our best models listed above are robust in the sense that they predict consistent values for the $n\left(\mathrm{H}^{0}\right)$ to within $5 \%$, and electron densities to within $25 \%$.

The radiation field incident on the LIC for Model 26 is shown in Fig. 1 and the spectral characteristics of the field for each model are listed in Table 5. The wavelengths regions $\lambda \leq 912 \AA$ and $\lambda \sim 1500 \AA$ are of primary importance for the photoionization of the cloud, the former because it determines $\mathrm{H}^{0}$ and $\mathrm{He}^{0}$ ionization, and the latter because it determines $\mathrm{Mg}^{0}$ ionization.

The ionization parameter is defined as $U \equiv \Phi / n(\mathrm{H}) c$, where $\Phi$ is the $\mathrm{H}$ ionizing photon flux, $n(\mathrm{H})$ is the total (neutral + ionized) hydrogen density at the cloud surface and $c$ is 
Table 3. Model column density results.

\begin{tabular}{cccccccc}
\hline \hline Model $^{a}$ & $\log N\left(\mathrm{H}_{\text {tot }}\right)$ & $\log N($ Ar I $)$ & $\log N($ Ar II $)$ & $\log N(\mathrm{Si} \mathrm{III})$ & $\frac{N(\mathrm{Mg} I \mathrm{II})}{N(\mathrm{Mg} \mathrm{I}}$ & $\frac{N(\mathrm{C} I \mathrm{II})}{N(\mathrm{C} \mathrm{II})}$ & $\frac{N(\mathrm{HI})}{N(\mathrm{He})}$ \\
Obs. $^{b}$ & - & - & - & 12.40 & $443_{-110}^{+197}$ & $93-430^{c}$ & $12-16$ \\
\hline 1 & 17.58 & 11.48 & 11.76 & 8.895 & 706.3 & 213.0 & 11.36 \\
2 & 17.78 & 11.67 & 11.95 & 9.641 & 325.3 & 234.1 & 11.40 \\
3 & 17.65 & 11.33 & 11.84 & 9.844 & 246.1 & 182.1 & 11.44 \\
4 & 17.82 & 11.55 & 12.00 & 10.12 & 136.5 & 198.0 & 11.80 \\
5 & 17.58 & 11.49 & 11.75 & 8.961 & 717.8 & 230.0 & 11.62 \\
6 & 17.78 & 11.67 & 11.94 & 9.711 & 286.7 & 244.9 & 11.62 \\
7 & 17.64 & 11.34 & 11.81 & 9.909 & 207.4 & 194.8 & 12.47 \\
8 & 17.82 & 11.56 & 11.97 & 10.17 & 119.7 & 208.2 & 12.66 \\
9 & 17.59 & 11.46 & 11.75 & 9.289 & 553.0 & 239.5 & 12.27 \\
10 & 17.78 & 11.66 & 11.93 & 9.861 & 210.3 & 248.1 & 12.14 \\
11 & 17.64 & 11.33 & 11.78 & 9.993 & 171.2 & 201.7 & 13.44 \\
12 & 17.82 & 11.54 & 11.95 & 10.23 & 104.3 & 210.6 & 13.52 \\
13 & 17.60 & 11.44 & 11.78 & 9.100 & 768.9 & 255.1 & 11.52 \\
$\mathbf{1 4}$ & 17.79 & 11.64 & 11.96 & 9.738 & 336.4 & 274.4 & 11.47 \\
15 & 17.67 & 11.30 & 11.85 & 9.944 & 251.2 & 215.9 & 11.65 \\
16 & 17.84 & 11.51 & 12.01 & 10.21 & 140.6 & 232.1 & 12.01 \\
17 & 17.58 & 11.47 & 11.76 & 8.926 & 840.1 & 262.5 & 11.65 \\
18 & 17.79 & 11.65 & 11.95 & 9.792 & 308.9 & 286.3 & 11.64 \\
19 & 17.66 & 11.30 & 11.82 & 10.02 & 208.5 & 229.2 & 12.76 \\
20 & 17.84 & 11.52 & 11.98 & 10.26 & 123.5 & 242.1 & 12.95 \\
21 & 17.59 & 11.43 & 11.76 & 9.318 & 633.1 & 280.8 & 12.41 \\
22 & 17.79 & 11.63 & 11.94 & 9.926 & 224.9 & 288.4 & 12.25 \\
23 & 17.66 & 11.29 & 11.79 & 10.10 & 175.0 & 235.5 & 13.81 \\
24 & 17.84 & 11.50 & 11.96 & 10.31 & 110.2 & 245.5 & 13.85 \\
25 & 17.66 & 11.32 & 11.84 & 9.887 & 271.3 & 213.6 & 11.71 \\
$\mathbf{2 6}$ & 17.74 & 11.56 & 11.91 & 9.676 & 383.9 & 270.1 & 11.70 \\
$\mathbf{2 7}$ & 17.63 & 11.36 & 11.79 & 9.751 & 331.1 & 242.0 & 12.51 \\
$\mathbf{2 8}$ & 17.73 & 11.59 & 11.90 & 9.625 & 411.6 & 284.5 & 11.79 \\
$\mathbf{2 9}$ & 17.62 & 11.38 & 11.77 & 9.720 & 329.3 & 251.6 & 13.01 \\
$\mathbf{3 0}$ & 17.72 & 11.60 & 11.88 & 9.642 & 386.6 & 295.1 & 12.08 \\
$\mathbf{4 2}$ & 17.77 & 11.70 & 11.92 & 9.555 & 482.9 & 317.9 & 11.51 \\
\hline
\end{tabular}

${ }^{a}$ The best models, consistent with all observational data (see Sect. 4), are indicated by bold face.

${ }^{b}$ Observational results from Gry \& Jenkins (2001) (see Table 1). The values listed for $N(\mathrm{HI}) / N(\mathrm{He} \mathrm{I})$ are the range of values observed excluding Feige 24 which is one of the most distant stars observed by Dupuis et al. (1995) and has unusually large $N(\mathrm{HI})$ and ratio values.

${ }^{c}$ The upper limit on $N(\mathrm{C}$ II) is not well determined observationally because of the saturation of the line. Gry \& Jenkins (2001) define it by assuming a solar C/S abundance ratio and using the observed S II column density. We find (see Slavin \& Frisch 2006) that the abundance of $\mathrm{C}$ required to match the $N\left(\mathrm{C} \mathrm{II}^{*}\right)$ observations is supersolar, with $\mathrm{C} / \mathrm{S} \sim 36-44$, which results in a much higher upper limit on $N(\mathrm{C}$ II $)$.

the speed of light. The total ionizing photon fluxes at the cloud surface (photons $\mathrm{cm}^{-2} \mathrm{~s}^{-1}$ ) for the three bands 13.6-24.6, 24.6-54.4, and 54.4-100 eV, are given by $\Phi_{\mathrm{H}}, \Phi_{\mathrm{He}^{0}}$, and $\Phi_{\mathrm{He}^{+}}$, respectively. The ratio of the total number of $\mathrm{H}^{0}$ and $\mathrm{He}^{0}$ ionizing photons in the incident radiation field is given by $Q\left(\mathrm{He}^{0}\right) / Q\left(\mathrm{H}^{0}\right)$. The quantity $\langle E\rangle(\mathrm{eV})$ is the mean energy of an ionizing photon, equal to the integrated energy flux from 13.6 to $100 \mathrm{eV}$ divided by the integrated photon flux over the same energy range.

In Fig. 3 we show $n\left(\mathrm{He}^{0}\right)$ and temperature of the CHISM for our model calculations. In Fig. 4 we show N II/N I vs. Mg II/Mg I, illustrating an anti-correlation of the ratios caused by the fact that $\mathrm{Mg}$ II $/ \mathrm{Mg}$ I decreases with electron density while N II/N I indicates the ionization level in the cloud. The ionization of the CHISM is listed in Table 6 for Model 26, where commonly observed elements are listed. The abundances of $\mathrm{He}, \mathrm{Ne}, \mathrm{Na}, \mathrm{Al}, \mathrm{P}$, $\mathrm{Ar}$, and $\mathrm{Ca}$ were assumed, based on solar abundances, and were not adjusted in the modeling process. The abundances of $\mathrm{C}, \mathrm{N}$, $\mathrm{O}, \mathrm{Mg}, \mathrm{Si}, \mathrm{S}$ and $\mathrm{Fe}$ were adjusted for each model to match observed column densities towards $\epsilon$ CMa (see Sect. 2). The elemental abundances that have been assumed, with the exception of that for $\mathrm{He}$, are not expected to have any significant impact on the model results.

\section{Discussion}

\subsection{Heliosphere boundary conditions}

As discussed above, the best models of those we calculated are determined by the match to the inflowing $\mathrm{He}^{0}$ density and temperature found by the in situ Ulysses measurements (Sect. 2.2), combined with the matching the LIC component column density ratios $\mathrm{Mg} \mathrm{II} / \mathrm{Mg}$ I and $\mathrm{CII} / \mathrm{C} \mathrm{II}^{*}$. These models span a fairly wide range in the model parameters: $n_{\mathrm{H}}=$ $0.213-0.232 \mathrm{~cm}^{-3}, \log T_{\mathrm{h}}=5.9-6.1 \mathrm{~K}, B_{0}=0.05-3.8 \mu \mathrm{G}$, and $N(\mathrm{HI})=3.0 \times 10^{17}-4.5 \times 10^{17} \mathrm{~cm}^{-2}$. Despite this, the predicted values for neutral $\mathrm{H}$ density and electron density in the CHISM lie within a remarkably narrow range: $n\left(\mathrm{H}^{0}\right)=0.19-0.20 \mathrm{~cm}^{-3}$, $n_{\mathrm{e}}=0.05-0.08 \mathrm{~cm}^{-3}$, for models that include the conductive boundary. For the one model without evaporation that is consistent with the data we find $n\left(\mathrm{H}^{0}\right)=0.186 \mathrm{~cm}^{-3}, n_{\mathrm{e}}=0.052 \mathrm{~cm}^{-3}$. Including the PUI Ne data as a constraint narrows the density results to $n\left(\mathrm{H}^{0}\right) \approx 0.19 \mathrm{~cm}^{-3}$ and $n_{\mathrm{e}}=0.06-0.07 \mathrm{~cm}^{-3}$. For these densities to stray out of this range would appear to require significant errors in the underlying comparison data, e.g. $n\left(\mathrm{He}^{0}\right)$ in the CHISM, or substantial non-equilibrium ionization effects in the LIC. The variation in the interstellar radiation field between the different models that match the data give us some degree 
Table 4. Model results for solar location.

\begin{tabular}{|c|c|c|c|c|c|c|c|c|c|c|c|}
\hline $\begin{array}{l}\text { Model }^{a} \\
\text { Obs. }^{b}\end{array}$ & $X(\mathrm{H})$ & $X(\mathrm{He})$ & $n\left(\mathrm{H}^{0}\right)$ & $\begin{array}{c}n\left(\mathrm{He}^{0}\right) \\
0.015\end{array}$ & $\begin{array}{c}n\left(\mathrm{~N}^{0}\right) \\
5.5 \times 10^{-6}\end{array}$ & $\begin{array}{c}n\left(\mathrm{O}^{0}\right) \\
4.8 \times 10^{-5}\end{array}$ & $\begin{array}{c}n\left(\mathrm{Ne}^{0}\right) \\
5.8 \times 10^{-6}\end{array}$ & $\begin{array}{c}n\left(\mathrm{Ar}^{0}\right) \\
1.6 \times 10^{-7}\end{array}$ & $n_{\mathrm{e}}$ & $n_{\mathrm{p}}$ & $\begin{array}{c}T \\
6300\end{array}$ \\
\hline 1 & 0.176 & 0.299 & 0.318 & 0.0269 & $1.84 \times 10^{-5}$ & $1.48 \times 10^{-4}$ & $1.39 \times 10^{-5}$ & $3.47 \times 10^{-7}$ & 0.0800 & 0.0678 & 4310 \\
\hline 2 & 0.196 & 0.352 & 0.251 & 0.0201 & $9.63 \times 10^{-6}$ & $7.71 \times 10^{-5}$ & $8.51 \times 10^{-6}$ & $2.69 \times 10^{-7}$ & 0.0724 & 0.0611 & 6450 \\
\hline 3 & 0.286 & 0.404 & 0.230 & 0.0191 & $1.34 \times 10^{-5}$ & $1.07 \times 10^{-4}$ & $7.81 \times 10^{-6}$ & $1.82 \times 10^{-7}$ & 0.106 & 0.0922 & 6280 \\
\hline 4 & 0.259 & 0.428 & 0.229 & 0.0175 & $8.86 \times 10^{-6}$ & $6.95 \times 10^{-5}$ & $6.45 \times 10^{-6}$ & $1.92 \times 10^{-7}$ & 0.0937 & 0.0799 & 7560 \\
\hline 5 & 0.174 & 0.310 & 0.300 & 0.0249 & $1.72 \times 10^{-5}$ & $1.39 \times 10^{-4}$ & $1.13 \times 10^{-5}$ & $3.30 \times 10^{-7}$ & 0.0750 & 0.0631 & 4610 \\
\hline 6 & 0.197 & 0.363 & 0.242 & 0.0190 & $9.13 \times 10^{-6}$ & $7.44 \times 10^{-5}$ & $7.08 \times 10^{-6}$ & $2.61 \times 10^{-7}$ & 0.0707 & 0.0593 & 6770 \\
\hline 7 & 0.278 & 0.441 & 0.225 & 0.0173 & $1.33 \times 10^{-5}$ & $1.04 \times 10^{-4}$ & $5.75 \times 10^{-6}$ & $1.78 \times 10^{-7}$ & 0.101 & 0.0866 & 6670 \\
\hline 8 & 0.257 & 0.459 & 0.223 & 0.0161 & $8.65 \times 10^{-6}$ & $6.79 \times 10^{-5}$ & $4.92 \times 10^{-6}$ & $1.89 \times 10^{-7}$ & 0.0918 & 0.0773 & 7860 \\
\hline 9 & 0.193 & 0.357 & 0.265 & 0.0209 & $1.53 \times 10^{-5}$ & $1.23 \times 10^{-4}$ & $7.52 \times 10^{-6}$ & $2.71 \times 10^{-7}$ & 0.0761 & 0.0634 & 5410 \\
\hline 10 & 0.203 & 0.392 & 0.235 & 0.0177 & $9.06 \times 10^{-6}$ & $7.24 \times 10^{-5}$ & $5.52 \times 10^{-6}$ & $2.43 \times 10^{-7}$ & 0.0723 & 0.0600 & 7230 \\
\hline 11 & 0.278 & 0.474 & 0.220 & 0.0158 & $1.29 \times 10^{-5}$ & $1.02 \times 10^{-4}$ & $4.42 \times 10^{-6}$ & $1.69 \times 10^{-7}$ & 0.0999 & 0.0847 & 7020 \\
\hline 12 & 0.262 & 0.490 & 0.219 & 0.0148 & $8.41 \times 10^{-6}$ & $6.66 \times 10^{-5}$ & $3.83 \times 10^{-6}$ & $1.78 \times 10^{-7}$ & 0.0928 & 0.0776 & 8160 \\
\hline 13 & 0.201 & 0.333 & 0.230 & 0.0191 & $1.34 \times 10^{-5}$ & $1.08 \times 10^{-4}$ & $8.94 \times 10^{-6}$ & $2.32 \times 10^{-7}$ & & & 4720 \\
\hline 14 & 0.215 & 0.376 & 0.193 & 0.0152 & $7.29 \times 10^{-6}$ & $5.95 \times 10^{-5}$ & $5.99 \times 10^{-6}$ & $1.94 \times 10^{-7}$ & 0.0 & 0.0 & 6650 \\
\hline 15 & 0.310 & 0.436 & 0.176 & 0.0143 & $1.02 \times 10^{-5}$ & $8.18 \times 10^{-5}$ & $5.35 \times 10^{-6}$ & $1.28 \times 10^{-7}$ & 0.0 & & 6470 \\
\hline 16 & 0.284 & 0.461 & 0.175 & 0.0131 & $6.76 \times 10^{-6}$ & $5.36 \times 10^{-5}$ & $4.40 \times 10^{-6}$ & $1.35 \times 10^{-7}$ & 0.0812 & 0.0695 & 7750 \\
\hline 17 & 0.178 & 0.315 & 0.255 & 0.0211 & $1.47 \times 10^{-5}$ & $1.19 \times 10^{-4}$ & $9.51 \times 10^{-6}$ & 2.75 & 0.0656 & 0.0553 & 4360 \\
\hline 18 & 0.214 & 0.383 & 0.188 & 0.0146 & $7.16 \times 10^{-6}$ & $5.81>$ & $5.07 \times 10^{-6}$ & & 0.0610 & 0.0513 & 6900 \\
\hline 19 & 0.302 & 0.472 & 0.173 & 0.0129 & $1.01 \times 10^{-5}$ & $8.03 \times 10$ & $3.93 \times 10^{-6}$ & $0^{-7}$ & 0.0872 & 0.0748 & 6860 \\
\hline 20 & 0.282 & 0.491 & 0.172 & 0.0120 & $6.61 \times 10^{-6}$ & $5.26 \times 10^{-5}$ & $3.37 \times 10^{-6}$ & $1.33 \times 10^{-7}$ & 0.0802 & 0.0677 & 8050 \\
\hline 21 & 0.204 & 0.374 & 0.211 & 0.0164 & $1.20 \times 10^{-5}$ & $9.85 \times 10^{-5}$ & $5.54 \times 10^{-6}$ & $2.04 \times 10^{-7}$ & 0.0648 & 0.0541 & 5390 \\
\hline 22 & 0.221 & 0.414 & 0.184 & 0.0136 & $6.96 \times 10^{-6}$ & $5.69 \times 10^{-5}$ & $3.90 \times 10^{-6}$ & $1.78 \times 10^{-7}$ & 0.0628 & 0.0523 & 7350 \\
\hline 23 & 0.305 & 0.506 & 0.168 & 0.0117 & $9.91 \times 10^{-6}$ & $7.82 \times 10^{-5}$ & $2.97 \times 10^{-6}$ & $1.18 \times 10^{-7}$ & 0.0868 & 0.0737 & 7220 \\
\hline 24 & 0.286 & 0.520 & 0.169 & 0.0111 & $6.57 \times 10^{-6}$ & $5.16 \times 10^{-5}$ & $2.60 \times 10^{-6}$ & $1.25 \times 10^{-7}$ & 0.0808 & 0.0676 & 8310 \\
\hline 25 & 0.297 & 0.427 & 0.187 & 0.0151 & $1.09 \times 10^{-5}$ & $8.67 \times 10^{-5}$ & $5.75 \times 10^{-6}$ & $1.41 \times 10^{-7}$ & 0.0907 & 0.0789 & 6360 \\
\hline 26 & 0.224 & 0.385 & 0.192 & 0.0151 & $8.32 \times 10^{-6}$ & $6.66 \times 10^{-5}$ & $5.96 \times 10^{-6}$ & $1.83 \times 10^{-7}$ & 0.0653 & 0.0554 & 6320 \\
\hline 27 & 0.258 & 0.426 & 0.195 & 0.0149 & $1.13 \times 10^{-5}$ & $8.97 \times 10^{-5}$ & $5.03 \times 10^{-6}$ & $1.63 \times 10^{-7}$ & 0.0796 & 0.0677 & 6240 \\
\hline 28 & 0.212 & 0.376 & 0.194 & 0.0152 & $8.24 \times 10^{-6}$ & $6.73 \times 10^{-5}$ & $5.49 \times 10^{-6}$ & $1.95 \times 10^{-7}$ & 0.0622 & 0.0523 & 6350 \\
\hline 29 & 0.242 & 0.429 & 0.202 & 0.0150 & $1.17 \times 10^{-5}$ & $9.30 \times 10^{-5}$ & $4.53 \times 10^{-6}$ & $1.74 \times 10^{-7}$ & 0.0769 & 0.0646 & 6300 \\
\hline 30 & 0.203 & 0.379 & 0.197 & 0.0151 & $8.51 \times 10^{-6}$ & $6.81 \times 10^{-5}$ & $4.78 \times 10^{-6}$ & $2.01 \times 10^{-7}$ & 0.0605 & 0.0502 & 6500 \\
\hline 42 & 0.189 & 0.355 & 0.186 & 0.0145 & $6.91 \times 10^{-6}$ & $5.71 \times 10^{-5}$ & $4.60 \times 10^{-6}$ & $2.04 \times 10^{-7}$ & 0.0522 & 0.0433 & 6590 \\
\hline
\end{tabular}

${ }^{a}$ The best models (see Sect. 4) are indicated by bold face.

${ }^{b}$ Observational results from Gloeckler \& Geiss (2004), Gloeckler (2005, private communication) and Witte et al. (1996) (see Table 1 for uncertainties).

of confidence that these densities are not highly sensitive to the details of the radiation field. The range $n_{\mathrm{e}}=0.05-0.08 \mathrm{~cm}^{-3}$ corresponds to an electron plasma frequency of $2.0-2.5 \mathrm{kHz}$, which is the frequency of the mysterious weak radio emission detected beyond the termination shock in the outer heliosphere (Gurnett \& Kurth 2005; Mitchell et al. 2004).

\subsection{Hydrogen filtration factor}

Tracers of $\mathrm{H}^{0}$ inside of the termination shock, after filtration, include the H I Ly $\alpha$ backscattered radiation, H pickup ions, and the slowdown of the solar wind at distances beyond $5 \mathrm{AU}$ from mass-loading by $\mathrm{H}$ PUIs. The range of $n\left(\mathrm{H}^{0}\right)$ found above to best fit the combined heliospheric $n\left(\mathrm{He}^{0}\right)$ and LIC data towards $\epsilon \mathrm{CMa}, n\left(\mathrm{H}^{0}\right)=0.19-0.20 \mathrm{~cm}^{-3}$, represents the density of neutral interstellar $\mathrm{H}$ atoms outside of the heliosphere, and removed from heliospheric influences. The hydrogen filtration factor, $f_{\mathrm{H}}$, can be obtained from comparisons between these models and interstellar $\mathrm{H}^{0}$ densities at the termination shock as inferred from in situ observations of interstellar $\mathrm{H}$ inside of the heliosphere.

The accompanying papers in this special section provide estimates of the interstellar $\mathrm{H}^{0}$ density at the termination shock. The solar wind slows down due to mass loading by interstellar $\mathrm{H}$, yielding $n\left(\mathrm{H}^{0}\right)=0.09 \pm 0.01 \mathrm{~cm}^{-3}$ at the termination shock (Richardson et al. 2008). The density of $\mathrm{H}$ pickup ions observed by Ulysses is inferred at the termination shock, yielding $n\left(\mathrm{H}^{0}\right)=0.09 \pm 0.04 \mathrm{~cm}^{-3}$; models of $\mathrm{H}$ atoms traversing the heliosheath regions then yield for the CHISM $n\left(\mathrm{H}^{0}\right)=0.20 \pm$ $0.02 \mathrm{~cm}^{-3}$ and $n_{\mathrm{p}}=0.04 \pm 0.02 \mathrm{~cm}^{-3}$, or $n_{\mathrm{e}} \sim 0.05 \mathrm{~cm}^{-3}$ (Bzowski et al. 2008). The radial variation in the response of the interplanetary Ly $\alpha 1215 \AA$ backscattered radiation to the solar rotational modulation of the $\operatorname{Ly} \alpha$ "beam" that excites the florescence yields $n\left(\mathrm{H}^{0}\right) \sim 0.085-0.095 \mathrm{~cm}^{-3}$, depending on the heliosphere model (Pryor et al. 2008). From these $n\left(\mathrm{H}^{0}\right)$ values at the termination shock, we estimate that $43 \%-58 \%$ of the H-atoms successfully traverse the heliosheath region, or $f_{\mathrm{H}} \sim 0.43-0.58$. Müller et al. (2008) evaluate filtration using five different plasma-neutral models, and find a range of $f_{\mathrm{H}}=0.52-0.74$. A hydrogen filtration of $f_{\mathrm{H}}=0.55 \pm 0.03$ is consistent with both in situ data and radiative transfer models.

\subsection{Gas-phase abundances}

The LIC photoionization models are forced to match the observed set of column densities (Tables 1 and 3). The gas-phase abundances of most elements are treated as free parameters that can be varied in order to match observed column densities, so that the successful models yield elemental abundances for the LIC that are automatically corrected for unobserved $\mathrm{H}^{+}$ (Table 7). The exceptions are that $\mathrm{He}, \mathrm{Ne}$, and Ar abundances, being unconstrained by the observations toward $\epsilon \mathrm{CMa}$, are not adjusted but are assumed to be $10^{5} \mathrm{ppm}, 123 \mathrm{ppm}$ and $2.82 \mathrm{ppm}$, respectively. In the models, $N\left(\mathrm{C} \mathrm{II}^{*}\right)$ is a constraint on both the 
Table 5. Characteristics of the model radiation field.

\begin{tabular}{|c|c|c|c|c|c|c|}
\hline Model $^{a}$ & $\bar{U}$ & $\begin{array}{c}\phi_{\mathrm{H}} \\
\text { photons } \mathrm{cm}^{-2} \mathrm{~s}^{-1}\end{array}$ & $\begin{array}{c}\phi_{\mathrm{He}^{0}} \\
\text { photons } \mathrm{cm}^{-2} \mathrm{~s}^{-1}\end{array}$ & $\begin{array}{c}\phi_{\mathrm{He}^{+}} \\
\text {photons } \mathrm{cm}^{-2} \mathrm{~s}^{-1}\end{array}$ & $\bar{Q}\left(\mathrm{He}^{0}\right) / Q\left(\mathrm{H}^{0}\right)$ & $\begin{array}{l}\langle E\rangle \\
\mathrm{eV}\end{array}$ \\
\hline 1 & $2.0 \times 10^{-6}$ & $4.6 \times 10^{3}$ & $7.5 \times 10^{3}$ & $2.8 \times 10^{3}$ & 0.46 & 74.6 \\
\hline 2 & $2.0 \times 10^{-6}$ & $5.3 \times 10^{3}$ & $7.3 \times 10^{3}$ & $2.7 \times 10^{3}$ & 0.44 & 72.4 \\
\hline 3 & $3.1 \times 10^{-6}$ & $9.0 \times 10^{3}$ & $1.3 \times 10^{4}$ & $2.7 \times 10^{3}$ & 0.49 & 57.7 \\
\hline 4 & $3.0 \times 10^{-6}$ & $8.6 \times 10^{3}$ & $1.2 \times 10^{4}$ & $2.6 \times 10^{3}$ & 0.49 & 58.6 \\
\hline 5 & $2.0 \times 10^{-6}$ & $4.0 \times 10^{3}$ & $7.0 \times 10^{3}$ & $3.8 \times 10^{3}$ & 0.43 & 79.0 \\
\hline 6 & $2.1 \times 10^{-6}$ & $4.9 \times 10^{3}$ & $6.9 \times 10^{3}$ & $3.8 \times 10^{3}$ & 0.41 & 76.2 \\
\hline 7 & $3.2 \times 10^{-6}$ & $7.4 \times 10^{3}$ & $1.4 \times 10^{4}$ & $3.8 \times 10^{3}$ & 0.53 & 61.3 \\
\hline 8 & $3.1 \times 10^{-6}$ & $7.4 \times 10^{3}$ & $1.3 \times 10^{4}$ & $3.7 \times 10^{3}$ & 0.52 & 61.5 \\
\hline 9 & $2.2 \times 10^{-6}$ & $3.8 \times 10^{3}$ & $7.4 \times 10^{3}$ & $5.6 \times 10^{3}$ & 0.40 & 80.0 \\
\hline 10 & $2.3 \times 10^{-6}$ & $4.8 \times 10^{3}$ & $7.2 \times 10^{3}$ & $5.6 \times 10^{3}$ & 0.38 & 77.3 \\
\hline 11 & $3.5 \times 10^{-6}$ & $6.6 \times 10^{3}$ & $1.5 \times 10^{4}$ & $5.6 \times 10^{3}$ & 0.53 & 63.4 \\
\hline 12 & $3.4 \times 10^{-6}$ & $6.9 \times 10^{3}$ & $1.5 \times 10^{4}$ & $5.5 \times 10^{3}$ & 0.52 & 63.2 \\
\hline 13 & $2.3 \times 10^{-6}$ & $4.3 \times 10^{3}$ & $7.0 \times 10^{3}$ & $2.8 \times 10^{3}$ & 0.46 & 76.9 \\
\hline 14 & $2.4 \times 10^{-6}$ & $5.1 \times 10^{3}$ & $6.8 \times 10^{3}$ & $2.7 \times 10^{3}$ & 0.43 & 74.3 \\
\hline 15 & $3.7 \times 10^{-6}$ & $8.5 \times 10^{3}$ & $1.2 \times 10^{4}$ & $2.7 \times 10^{3}$ & 0.49 & 58.8 \\
\hline 16 & $3.6 \times 10^{-6}$ & $8.2 \times 10^{3}$ & $1.2 \times 10^{4}$ & $2.6 \times 10^{3}$ & 0.49 & 59.5 \\
\hline 17 & $2.3 \times 10^{-6}$ & $3.7 \times 10^{3}$ & $6.4 \times 10^{3}$ & $3.8 \times 10^{3}$ & 0.42 & 81.8 \\
\hline 18 & $2.4 \times 10^{-6}$ & $4.7 \times 10^{3}$ & $6.2 \times 10^{3}$ & $3.8 \times 10^{3}$ & 0.39 & 78.4 \\
\hline 19 & $3.9 \times 10^{-6}$ & $7.1 \times 10^{3}$ & $1.4 \times 10^{4}$ & $3.8 \times 10^{3}$ & 0.53 & 62.2 \\
\hline 20 & $3.8 \times 10^{-6}$ & $7.2 \times 10^{3}$ & $1.3 \times 10^{4}$ & $3.7 \times 10^{3}$ & 0.52 & 62.2 \\
\hline 21 & $2.6 \times 10^{-6}$ & $3.6 \times 10^{3}$ & $6.8 \times 10^{3}$ & $5.6 \times 10^{3}$ & 0.39 & 82.0 \\
\hline 22 & $2.7 \times 10^{-6}$ & $4.6 \times 10^{3}$ & $6.6 \times 10^{3}$ & $5.6 \times 10^{3}$ & 0.36 & 78.9 \\
\hline 23 & $4.2 \times 10^{-6}$ & $6.3 \times 10^{3}$ & $1.5 \times 10^{4}$ & $5.6 \times 10^{3}$ & 0.52 & 64.4 \\
\hline 24 & $4.2 \times 10^{-6}$ & $6.7 \times 10^{3}$ & $1.4 \times 10^{4}$ & $5.5 \times 10^{3}$ & 0.51 & 64.1 \\
\hline 25 & $3.5 \times 10^{-6}$ & $7.9 \times 10^{3}$ & $1.2 \times 10^{4}$ & $2.7 \times 10^{3}$ & 0.49 & 60.3 \\
\hline 26 & $2.5 \times 10^{-6}$ & $5.0 \times 10^{3}$ & $7.5 \times 10^{3}$ & $2.7 \times 10^{3}$ & 0.45 & 73.2 \\
\hline 27 & $3.1 \times 10^{-6}$ & $5.4 \times 10^{3}$ & $1.0 \times 10^{4}$ & $3.8 \times 10^{3}$ & 0.50 & 68.9 \\
\hline 28 & $2.4 \times 10^{-6}$ & $4.3 \times 10^{3}$ & $6.4 \times 10^{3}$ & $3.8 \times 10^{3}$ & 0.40 & 79.5 \\
\hline 29 & $3.0 \times 10^{-6}$ & $4.6 \times 10^{3}$ & $9.7 \times 10^{3}$ & $5.6 \times 10^{3}$ & 0.45 & 73.6 \\
\hline 30 & $2.4 \times 10^{-6}$ & $3.8 \times 10^{3}$ & $5.5 \times 10^{3}$ & $5.6 \times 10^{3}$ & 0.33 & 84.6 \\
\hline 42 & $2.2 \times 10^{-6}$ & $3.6 \times 10^{3}$ & $3.6 \times 10^{3}$ & $5.6 \times 10^{3}$ & 0.25 & 91.1 \\
\hline
\end{tabular}

${ }^{a}$ The best models (see Sect. 4) are indicated by bold face.

C abundance (in place of the heavily saturated C II $1335 \AA$ Aline) and $n_{\mathrm{e}}$, such that the product of the abundance and $n_{\mathrm{e}}$ is more tightly limited than either quantity individually. The requirement to match both $N\left(\mathrm{C} \mathrm{II}^{*}\right)$ and $n\left(\mathrm{He}^{0}\right)$ effectively restricts the ionization fraction of $\mathrm{H}$, which in turn limits $\mathrm{O}$ and $\mathrm{N}$ ionization whose ionization fractions are tied by charge-transfer to the $\mathrm{H}$ ionization at LIC temperatures.

Early studies showed that the abundances of refractory elements in the very local ISM are enhanced compared to abundances in cold disk gas (Marschall \& Hobbs 1972; Stokes 1978; Frisch 1981). Throughout warm and cold disk gas, the underabundances of refractory elements compared to solar abundances (by factors of $10^{-1}-10^{-4}$ ) are taken to represent depletion onto interstellar dust grains (e.g. Savage \& Sembach 1996). This view is supported by the correlation found between elemental depletions and the temperature characteristic of condensation at solar pressure and composition (Ebel 2000), and assumes that there is a reference abundance pattern that characterizes the cloud, and remains constant over the cloud lifetime as atoms are exchanged between the gas and dust phases. Below (Sect. 5.4) we compare solar abundances with observed gas-phase abundances to predict the gas-to-dust mass ratios for the LIC, based on the assumption that LIC gas and dust have remained coupled over the cloud lifetime.

An important question is whether the LIC has solar abundances. Isotopes of ${ }^{18} \mathrm{O}$ and ${ }^{22} \mathrm{Ne}$ isotopes measured in the anomalous cosmic ray population suggest that this is so. ACRs are characterized by a rising particle flux for energies below $10-50 \mathrm{MeV} /$ nucleon, and this characteristic spectral signature is seen for ${ }^{16} \mathrm{O},{ }^{18} \mathrm{O},{ }^{20} \mathrm{Ne}$, and ${ }^{22} \mathrm{Ne}$. Ratios of ${ }^{16} \mathrm{O} /{ }^{18} \mathrm{O} \sim 500$ and ${ }^{20} \mathrm{Ne} /{ }^{22} \mathrm{Ne} \sim 13.7$ are found for both the ACRs and solar material, indicating that the CHISM and solar material have similar compositions (Leske et al. 2000; Leske 2000). We therefore adopt solar abundances as the underlying reference abundance pattern for the LIC.

Unfortunately a prominent uncertainty exists in the correct solar abundances of volatile elements such as $\mathrm{O}$ and $\mathrm{S}$, which have low condensation temperatures ( $T_{\text {cond }}, 180 \mathrm{~K}$ and $700 \mathrm{~K}$ respectively), and noble elements such as $\mathrm{Ne}$ and Ar. Solar abundances are determined from photospheric data $(\mathrm{C}, \mathrm{N}, \mathrm{O}, \mathrm{Mg}$, $\mathrm{Si}, \mathrm{S}, \mathrm{Fe})$, the solar wind ( $\mathrm{Ar}, \mathrm{Ne})$, solar active regions $(\mathrm{Ne})$, and helioseismic data (He); abundances of non-volatile elements are also found from meteoritic data (Grevesse \& Sauval 1998; Holweger 2001; Lodders 2003; Grevesse et al. 2007). Solar abundances from these studies are listed in Table 8. Our results, discussed below, indicate that if the LIC has a solar abundance composition, as indicated by the ${ }^{18} \mathrm{O}$ and ${ }^{22} \mathrm{Ne}$ data, then the lower abundances found by Grevesse et al. (2007) are preferred by our models.

Ne: in these models we have assumed the Ne abundance is 123 ppm (Anders \& Grevesse 1989), which is based on a combination of photospheric and interstellar data. Solar system 


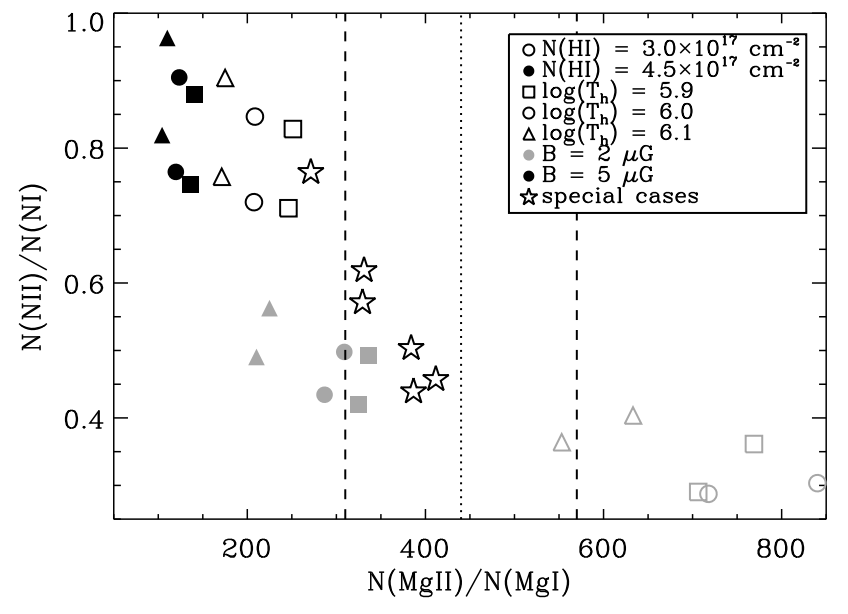

Fig. 4. Model results for $N(\mathrm{~N}$ II $) / N(\mathrm{~N}$ I) versus $\mathrm{Mg}$ II $/ \mathrm{Mg}$ I. The symbols have the same meaning as in Fig. 3. In this case the models with higher $n\left(\mathrm{H}^{0}\right)$ lie below and slightly to the left of those with lower density. $N(\mathrm{~N} \mathrm{II}) / N(\mathrm{~N} \mathrm{I})$ is an indicator of cloud ionization fraction while $\mathrm{Mg}$ II $/ \mathrm{Mg}$ I goes as $1 / n_{\mathrm{e}}$. The dotted line is the observed value for $\mathrm{Mg}$ II/Mg I and the dashed lines indicate the $1-\sigma$ error range for the value. We see that models that match the observed ratio all correspond to relatively low ionization, $X(\mathrm{H}) \sim 0.19-0.26$ in the CHISM.

Table 6. Model 26 results for ionization fractions ${ }^{a}$.

\begin{tabular}{llllll}
\hline \hline Element & PPM & I & II & III & IV \\
\hline $\mathrm{H}$ & $10^{6}$ & 0.776 & 0.224 & - & - \\
$\mathrm{He}$ & $10^{5}$ & 0.611 & 0.385 & $4.36(-3)$ & - \\
$\mathrm{C}$ & 661 & $2.68(-4)$ & 0.975 & 0.0244 & 0.000 \\
$\mathrm{~N}$ & 46.8 & 0.720 & 0.280 & $8.52(-5)$ & 0.000 \\
$\mathrm{O}$ & 331 & 0.814 & 0.186 & $4.71(-5)$ & 0.000 \\
$\mathrm{Ne}$ & 123 & 0.196 & 0.652 & 0.152 & $2.79(-6)$ \\
$\mathrm{Na}$ & 2.04 & $1.47(-3)$ & 0.843 & 0.155 & $6.34(-6)$ \\
$\mathrm{Mg}$ & 6.61 & $1.98(-3)$ & 0.850 & 0.148 & 0.000 \\
$\mathrm{Al}$ & 0.0794 & $5.37(-5)$ & 0.976 & 0.0118 & 0.0123 \\
$\mathrm{Si}$ & 8.13 & $4.21(-5)$ & 0.999 & $8.02(-4)$ & $3.10(-5)$ \\
$\mathrm{P}$ & 0.219 & $1.35(-4)$ & 0.977 & 0.0232 & $9.29(-5)$ \\
$\mathrm{S}$ & 15.8 & $6.47(-5)$ & 0.971 & 0.0288 & $1.95(-6)$ \\
$\mathrm{Ar}$ & 2.82 & 0.263 & 0.500 & 0.238 & $2.83(-6)$ \\
$\mathrm{Ca}$ & $4.07(-4)$ & $9.21(-6)$ & 0.0155 & 0.984 & $1.87(-4)$ \\
$\mathrm{Fe}$ & 2.51 & $7.01(-5)$ & 0.975 & 0.0245 & $5.75(-6)$ \\
\hline
\end{tabular}

${ }^{a}$ Numbers less than $10^{-3}$ are written as $x(y)$ where $y$ is the exponent and $x$ is the mantissa (or significand).

$\mathrm{Ne}$ abundances are difficult to measure because of FIP effects, however values include $77 \mathrm{ppm}$ (Grevesse et al. 2007, after adding 0.05 dex to account for gravitational settling of the elements), and $\sim 41 \mathrm{ppm}$ for solar wind in coronal holes (Gloeckler $\&$ Geiss 2007). The predicted densities of $n\left(\mathrm{Ne}^{0}\right)$ in the CHISM for models 26 and 28 (Table 4) are in agreement with the most recent PUI results for $\mathrm{Ne}$ (Table 1), and $\mathrm{Ne}$ densities as low as $\sim 100 \mathrm{ppm}$ are allowed when filtration is included.

The CHISM Ne abundances indicated by these results appear to be consistent with $\mathrm{Ne}$ abundances and ionization levels in the global ISM. The Ne abundance in the Orion nebula is $100 \mathrm{ppm}$ (Simpson et al. 2004). Takei et al. (2002) measured X-ray absorption edges formed by $\mathrm{Ne}$ and $\mathrm{O}$ in the interstellar gas and dust towards Cyg X-2, and found abundances of $\mathrm{Ne} / \mathrm{H} \sim 92 \mathrm{ppm}$ and $\mathrm{O} / \mathrm{H} \sim 579 \mathrm{ppm}$ when both atomic and compound forms in the sightline were included. Juett et al. (2006) observed the $\mathrm{X}$-ray absorption edges of $\mathrm{Ne}$ and $\mathrm{O}$ towards nine X-ray binaries which sampled both neutral and ionized warm material, and
Table 7. Elemental gas phase abundances (ppm).

\begin{tabular}{crrrrrrr}
\hline \hline & \multicolumn{7}{c}{ Element } \\
\cline { 2 - 7 } Model No. & $\mathrm{C}$ & $\mathrm{N}$ & $\mathrm{O}$ & $\mathrm{Mg}$ & $\mathrm{Si}$ & $\mathrm{S}$ & $\mathrm{Fe}$ \\
\hline 14 & 589 & 40.7 & 295 & 5.89 & 7.24 & 14.1 & 2.24 \\
21 & 955 & 60.3 & 447 & 9.77 & 11.5 & 22.9 & 3.55 \\
25 & 631 & 66.1 & 437 & 7.76 & 10.0 & 19.5 & 3.09 \\
26 & 661 & 46.8 & 331 & 6.61 & 8.13 & 15.8 & 2.51 \\
27 & 759 & 64.6 & 437 & 8.71 & 10.7 & 20.9 & 3.31 \\
28 & 708 & 45.7 & 331 & 7.08 & 8.32 & 16.6 & 2.57 \\
29 & 813 & 64.6 & 437 & 9.33 & 11.0 & 21.9 & 3.39 \\
30 & 741 & 46.8 & 331 & 7.41 & 8.51 & 17.0 & 2.63 \\
42 & 724 & 39.8 & 295 & 6.76 & 7.76 & 15.1 & 2.34 \\
\hline
\end{tabular}

Table 8. Solar abundances (ppm).

\begin{tabular}{lllll}
\hline \hline & $\begin{array}{l}\text { Grevesse } \\
\text { Sauval } \\
(1998)\end{array}$ & Holweger & Lodders & $\begin{array}{l}\text { Grevesse } \\
\text { et al. } \\
(2007)^{a}\end{array}$ \\
\hline $\mathrm{C}$ & $334 \pm 46$ & $391(+110,-86)$ & $290 \pm 27$ & $275 \pm 34$ \\
$\mathrm{~N}$ & $84 \pm 12$ & $85.3_{-19}^{+25}$ & $82 \pm 20$ & $67 \pm 10$ \\
$\mathrm{O}$ & $683 \pm 94$ & $545_{-90}^{+107}$ & $579 \pm 66$ & $513 \pm 63$ \\
$\mathrm{Ne}$ & $121 \pm 17$ & $100_{-15}^{+17}$ & $91 \pm 21$ & $77 \pm 12$ \\
$\mathrm{Mg}$ & $38 \pm 4$ & $34.5_{-4.5}^{+5.1}$ & $41 \pm 2$ & $38 \pm 9$ \\
$\mathrm{Si}$ & $35 \pm 4$ & $34.4_{-3.7}^{+4.1}$ & $41 \pm 2$ & $36 \pm 4$ \\
$\mathrm{~S}$ & $22 \pm 5$ & - & $18 \pm 2$ & $15 \pm 2$ \\
$\mathrm{Ar}$ & $2.54 \pm 0.35$ & - & $4.24 \pm 0.77$ & $1.70 \pm 0.34$ \\
$\mathrm{Fe}$ & $32 \pm 4$ & $28.1_{4.8}^{5.8}$ & $35 \pm 2$ & $32 \pm 4$ \\
\hline
\end{tabular}

a Protosolar abundances are obtained by increasing the photospheric abundances by 0.05 dex for elements heavier than $\mathrm{He}$, as suggested by Grevesse et al. (2007).

found that the ionized states formed in the ionized material have the ratio $\mathrm{Ne}$ III/Ne II $\sim 0.23$. This value is identical to the predictions of model $26, \mathrm{Ne}$ III/Ne II $\sim 0.23$, a fortuitous agreement that may indicate that the EUV radiation field in the CHISM is similar to the generic galactic EUV field in the solar vicinity.

Ar: solar Ar abundance determinations range between $\mathrm{Ar} / \mathrm{H} \sim 1.4-5.0 \mathrm{ppm}$ (Table 8); we have assumed $\mathrm{Ar} / \mathrm{H}=$ $2.82 \mathrm{ppm}$. The predicted Ar density at the Sun is within the uncertainties of the PUI data, although the range of possible filtration factors (0.64-0.95, see Sect. 2.2) also allow considerable leeway.

O: in the warm ISM such as the LIC, the ionization of oxygen and hydrogen is tightly coupled over timescales of $\sim 100$ years by charge transfer (Field \& Steigman 1971), so that the assumed $N(\mathrm{HI})$ combined with $N(\mathrm{OI})$ measurements act to constrain the deduced $\mathrm{O}$ abundance in the gas. The two best models $(26,28)$ correspond to $\mathrm{O} / \mathrm{H}=331 \mathrm{ppm}$, however the $\mathrm{O}$ column density measurements are based on the saturated $1302 \AA$ line, and have $\sim 35 \%$ uncertainty. The modeled LIC value of $\mathrm{O} / \mathrm{H} \sim 331 \mathrm{ppm}$ indicates that $\sim 35 \%$ of the $\mathrm{O}$ atoms are depleted onto dust grains. An oxygen filtration factor of $f_{\mathrm{O}} \sim 0.75$ is required by the PUI data and Model 26.

These models yield gas-phase $\mathrm{O}$ abundances that are consistent with observations of more distant interstellar sightlines. The ratio $N(\mathrm{O} \mathrm{I}) / N(\mathrm{HI})$ is measured in both low and high extinction clouds. Oliveira et al. (2003) used unsaturated O I lines in the 910-1100 $\AA$ interval and found O I/H I = $317 \pm 19$ ppm for $\sim 30$ sightlines that included both types of material. Sightlines with detected $\mathrm{H}_{2}, N(\mathrm{H})>10^{20.5} \mathrm{~cm}^{-2}$ and $\left\langle n_{\mathrm{H}}\right\rangle=0.1-3.3 \mathrm{~cm}^{-3}$, yield $\mathrm{O} / \mathrm{H}=319 \pm 14 \mathrm{ppm}$ (Meyer et al. 1997). A survey of 19 stars with an average distance of $2.6 \mathrm{kpc}$ by André et al. (2003) found $\mathrm{OI} / \mathrm{HI}=408 \pm 14 \mathrm{ppm}$, where the long sightline and high average value $N(\mathrm{H}) / E(B-V)=6.3 \times 10^{21} \mathrm{~cm}^{-2} \mathrm{mag}^{-1}$ 
indicate a bias towards sightlines containing many clouds that individually have low extinctions. A larger sample of 56 sightlines for a range of extinctions and distances show that sightlines with higher average mean densities, $\left\langle n_{\mathrm{H}}\right\rangle$, show $\mathrm{O} / \mathrm{H}=284 \pm 12 \mathrm{ppm}$, versus $\mathrm{O} / \mathrm{H}=390 \pm 10 \mathrm{ppm}$ for stars with low values of $\left\langle n_{\mathrm{H}}\right\rangle$ (Cartledge et al. 2004). For comparison, solar abundance studies yield a range of $\sim 450-780 \mathrm{ppm}$.

C: the best Models 26 and 28 yield a gas-phase abundance of $\mathrm{C}$ of $\mathrm{C} / \mathrm{H}=661$ and 708 compared to solar abundances of $\sim 240-500 \mathrm{ppm}$, which is consistent with our earlier results (Slavin \& Frisch 2006) indicating an overabundance of $C$ in the LIC. We speculate that shock destruction of carbonaceous grains, perhaps combined with some local spatial decoupling between carbonaceous and silicate grains, may explain these findings.

Singly-ionized carbon is an important coolant in the LIC (Sect. 5.5), so the $\mathrm{C}$ overabundance is required to maintain the temperature of the CHISM at the observed value. The carbon abundance obtained here indirectly depends on the $\mathrm{Mg}$ II $\rightarrow \mathrm{Mg}$ I dielectronic recombination coefficient that determines the ratio $\mathrm{Mg} \mathrm{II} / \mathrm{Mg}$ I, since that ratio is used as a criteria for the best models. The same ionization correction that gives the $\mathrm{C}$ abundance also successfully predicts $\mathrm{Ne}$ ionization in global warm partially ionized medium and the $\mathrm{S}$ abundances in the LIC, although this may be a fortuitous coincidence. In the adjacent sightline towards Sirius the LIC has $N(\mathrm{C}$ II $) / N(\mathrm{HI})=1050 \pm 850 \mathrm{ppm}$ (Hébrard et al. 1999). An ionization correction of $\sim 300 \%$ is indicated to make this value consistent with solar abundances, and such a large ionization correction is not consistent with the ionization levels of $X(\mathrm{H}) \sim 20-26 \%$ found here. In contrast, sightlines with cold ISM show $\mathrm{C}$ abundances on the order of $135 \pm 46$ ppm (Sofia et al. 1997; Sembach et al. 2000).

$\mathbf{N}$ : the best models (26 and 28) find $\mathrm{N} / \mathrm{H}=46-47 \mathrm{ppm}$, compared to solar values of $\sim 57-110 \mathrm{ppm}$. These results are consistent with the PUI results, $\mathrm{N} / \mathrm{H} \sim 19-47 \mathrm{ppm}$, after filtration factor uncertainties are included. The $\mathrm{N}$ and $\mathrm{O}$ results and favor an ISM abundance pattern for volatiles similar to the Grevesse et al. (2007) photospheric abundances.

S: the best models predict $\mathrm{S} / \mathrm{H}=16-17$, compared to solar values of 13-27 (including uncertainties, see Table 8). Sulfur is found to have little or no depletion onto dust grains in warm diffuse ISM (e.g. Welty et al. 1999).

Mg, Si, Fe: these refractory elements are observed in the LIC gas with abundances far below solar (factors of 3-15). Approximately $92 \%, 82 \%$, and $77 \%$ of the $\mathrm{Fe}, \mathrm{Mg}$, and $\mathrm{Si}$, respectively, are presumably depleted onto interstellar dust grains. If (Grevesse et al. 2007) abundances are assumed for the LIC, then to within the uncertainties the LIC dust has the relative composition of $\mathrm{Fe}: \mathrm{Mg}: \mathrm{Si}: \mathrm{O}=1: 1: 1: 4$, as is consistent with amorphous olivines $\mathrm{MgFeSiO}_{4}$. Fe and $\mathrm{Si}$ are dominantly singly ionized, while $\mathrm{Mg}$ has a significant fraction $(\sim 15 \%)$ that is twice ionized. The gas-phase abundances of these refractory elements are highly subsolar, even after ionization corrections are made, indicating that these elements are substantially depleted onto interstellar dust grains. In contrast to $\mathrm{C}$, however, the silicate dust in the LIC that carries the missing $\mathrm{Mg}, \mathrm{Si}$, and $\mathrm{Fe}$ has experienced far less destruction than the carbonaceous grains.

Ca II, Na I: weak lines of the trace ionization species Ca II and $\mathrm{Na} \mathrm{I}$ are common diagnostics of ionization and abundance for interstellar clouds, including the partially ionized LIC; Na I is also frequently used as a diagnostic of the $\mathrm{H}$ column density. We note that our models show that the ratios $N(\mathrm{Ca}$ II $) / N(\mathrm{Na} \mathrm{I})$, $N(\mathrm{Na} \mathrm{I}) / N(\mathrm{H})$, and $N(\mathrm{NaI}) / N(\mathrm{HI})$ vary by $30 \%, 77 \%$, and $93 \%$, respectively, between the best models (Models 26-30, 42). As
Table 9. Gas-to-dust mass ratios from models and in situ observations.

\begin{tabular}{lccccccc}
\hline \hline & \multicolumn{7}{c}{ Model $^{a}$} \\
\cline { 2 - 8 } Source & 14 & 26 & 27 & 28 & 29 & 30 & 42 \\
\hline GS98 & 137 & 149 & 196 & 149 & 197 & 150 & 138 \\
Lodders & 158 & 174 & 238 & 174 & 239 & 175 & 160 \\
Grevesse & 194 & 217 & 321 & 217 & 323 & 218 & 196 \\
In situ & 115 & 116 & 123 & 116 & 125 & 116 & 107 \\
\hline
\end{tabular}

${ }^{a}$ The source of the comparison solar abundances is listed in Col. 1 Table 8. The in situ dust flux is from Landgraf et al. (2000), corrected downwards by $20 \%$ as recommended by Altobelli et al. (2004) to account for side-wall impacts.

trace ionization species, the densities of $\mathrm{Na}$ I and $\mathrm{Ca}$ II are highly sensitive to volume density, $n\left(\mathrm{Na}^{0}\right), n\left(\mathrm{Ca}^{+}\right) \propto n(\mathrm{H}) n_{\mathrm{e}}$. We therefore conclude that $\mathrm{NaI}$ and $\mathrm{Ca}$ II are imprecise diagnostics of ionization levels, $\mathrm{H}$ density, and abundances in warm partially ionized clouds.

\subsection{Gas-to-dust mass ratio}

Because the abundances are automatically corrected for unobserved $\mathrm{H}^{+}$, we use the model results to infer the total mass of the interstellar dust. We implicitly assume that the gas and dust in the LIC form a coupled and closed system that evolves together as the cloud moves through the LSR (with no decoupling of the gas and dust and no injection of dust) so that the total of gas phase and dust phase elemental abundances remain a constant, presumably close to standard solar abundances. The lifetime of the LIC, for which this assumption must hold is uncertain, but if the LIC has its origins in the Loop I/Scorpius-Centaurus superbubble, then given its LSR velocity of 16-21 pc/Myr, we would require that the LIC gas and dust remained a closed system over timescales of 4-5 Myr (Frisch \& Slavin 2006; Frisch 1981). Gas-to-dust mass ratios calculated from the best models (26 and 28) using the missing-mass argument ${ }^{2}$ are in the range $R_{\mathrm{G} / \mathrm{D}}=149-217$, depending on solar abundances. The detailed information about $R_{\mathrm{G} / \mathrm{D}}$ for different assumptions and the different models is listed in Table 9.

For comparison, $R_{\mathrm{G} / \mathrm{D}}$ determined from comparisons of in situ observations of interstellar dust inside of the solar system, compared to the gas densities of these models, yield $R_{\mathrm{G} / \mathrm{D}}=115-125$ (Table 9, Landgraf et al. 2000; Altobelli et al. 2004). The in situ $R_{\mathrm{G} / \mathrm{D}}$ is an upper limit, since the smallest interstellar dust grains (radii $\leq 0.15 \mu \mathrm{m}$ ) with large charge-to-mass ratios (and thus small Larmor radii) are excluded from the heliosphere by the interstellar magnetic field which is draped over the heliosphere.

For all the models, the $R_{\mathrm{G} / \mathrm{D}}$ determined from comparing in situ dust measurements with the CHISM gas mass flux is lower than that determined by assuming solar abundances and using the gas phase abundances we determine to find $R_{\mathrm{G} / \mathrm{D}}$. This suggests that somehow the dust flowing into the heliosphere is concentrated relative to the gas, compared to the overall LIC sightline towards $\epsilon \mathrm{CMa}$. The lower solar abundances of Grevesse et al. (2007) result in lower required depletions, and produces stronger disagreements with $R_{\mathrm{G} / \mathrm{D}}$ determined from in situ data. We do not understand this result, which we have found previously (Frisch et al. 1999). Since $R_{\mathrm{G} / \mathrm{D}}$ is sensitive to the mass of $\mathrm{Fe}$ in the dust grains (Frisch \& Slavin 2003), we suggest that

\footnotetext{
2 This argument assumes that the ISM reference abundances, in this case solar abundances, represent the sum of the atoms in the gas plus the dust (Frisch et al. 1999).
} 
Table 10. Major heat sources in LIC Gas ${ }^{a}$.

\begin{tabular}{lc}
\hline \hline Source $^{b}$ & Fraction of heating \\
\hline H I & 0.657 \\
He I & 0.248 \\
Dust & 0.055 \\
He II & 0.016 \\
Cosmic rays & 0.010 \\
\hline
\end{tabular}

${ }^{a}$ Results for model 26. Other models are qualitatively the same, though there are some quantitative variations.

${ }^{b}$ For lines with ion names, the source here denotes the ion that is photoionized. Dust heating comes from photoelectric ejection by photons of the background FUV radiation field. Cosmic ray heating comes from electron impact ionization of the gas and direct heating of the electrons in the LIC plasma by the cosmic ray electrons.

Table 11. Major Coolants in LIC Gas ${ }^{a}$.

\begin{tabular}{|c|c|}
\hline Ion/Line & Fraction of cooling \\
\hline [C II] $157.6 \mu \mathrm{m}$ & 0.428 \\
\hline [S II] $6731 \AA$ & 0.145 \\
\hline Fe II (total) & 0.074 \\
\hline [Si II] $34.8 \mu \mathrm{m}$ & 0.065 \\
\hline$[\mathrm{Ne}$ II $] 12.8 \mu \mathrm{m}$ & 0.035 \\
\hline [O I] $63.2 \mu \mathrm{m}$ & 0.028 \\
\hline H recomb. & 0.024 \\
\hline Dust & 0.024 \\
\hline [Ne III] $15.6 \mu \mathrm{m}$ & 0.020 \\
\hline [N II] $6584 \AA$ & 0.018 \\
\hline Net free-free & 0.018 \\
\hline [O I] $6300 \AA$ & 0.017 \\
\hline [O II] $3727 \AA$ & 0.011 \\
\hline [Ar II] $6.98 \mu \mathrm{m}$ & 0.011 \\
\hline
\end{tabular}

${ }^{a}$ Results for model 26. Other models show similiar results.

this difference may indicate inhomogeneous mixing of the gas and silicate dust over the $\sim 0.64$ pc extent of the LIC.

\subsection{Heating and cooling rates}

The heating and cooling rates for Model 26 are listed in Tables 10 and 11 . The primary heat sources are photoelectrons from the ionization of $\mathrm{H}^{0}$ and $\mathrm{He}^{0}$, with dust and cosmic ray heating contributing less than $7 \%$ of the heating. The dominant source of cooling is the [C II] $157.6 \mu \mathrm{m}$ fine-structure line, making up $43 \%$ of the total. This is more than twice the contribution of any other coolant. Nearly all the cooling is due to optical and infrared forbidden lines with many lines contributing at the $\sim 1 \%$ level. H recombination, free-free emission and dust, through the capture of electrons onto grain surfaces, also contribute at about a $2 \%$ level. The importance of $\mathrm{C}$ II as both a constraint on the $\mathrm{C}$ abundance in our models as well as a major coolant means that any model that aims to reduce the abundance of $\mathrm{C}$ to a solar level faces severe difficulties. The models with LIC temperatures in the $T_{\mathrm{He}^{0}}=6300 \pm 340 \mathrm{~K}$ range indicated by the in situ $\mathrm{He}^{0}$ data all require supersolar abundances of $\mathrm{C}$. The total heating/cooling rate for the LIC at the Sun for this model is $3.55 \times 10^{-26} \mathrm{erg} \mathrm{cm}^{-3} \mathrm{~s}^{-1}$.

\subsection{Radiation field}

Recently it has been proposed that a significant portion of the soft X-ray background can be attributed to charge-transfer (a.k.a. charge exchange) between the solar wind ions (e.g., $\mathrm{O}^{+7}$ and $\mathrm{O}^{+8}$ ) and interstellar neutrals (Cravens 2000; Snowden et al. 2004; Wargelin et al. 2004; Smith et al. 2005; Koutroumpa et al. 2006). While it seems at present that some fraction of the low energy X-rays are from this mechanism, it is unclear how large that fraction is. We note that basing the properties of the local hot plasma in the galactic plane on SXRB emission at energies $E>0.3 \mathrm{keV}$ is problematical. (Bellm \& Vaillancourt 2005) have compared the Wisconsin B and Be band data with the ROSAT R12 data, and concluded that the observed anti-correlation between $\mathrm{R} 12$ and $N(\mathrm{HI})$ indicates that more than $34 \%$ of the SXRB generated in the Galactic disk must come from the Local Bubble. They also concluded that a heavily depleted plasma with $\log T \sim 5.8$ is consistent both with the McCammon et al. (2002); Sanders et al. (2001) X-ray spectral data, and the upper limits set on the EUV emission by CHIPS (Hurwitz et al. 2004). When the Robertson \& Cravens (2003) models of SXRB production by charge-transfer with the solar wind are considered, then only half of the SXRB in the plane is required to arise from a hot local plasma. We also note that the atomic physics for the calculation of the low energy part of the emission is still quite uncertain (Kharchenko, private communication). At this point we take the simple approach of ignoring the charge-transfer emission, though we plan to consider its possible impact in future work by reducing the assumed SXRB flux from hot gas. As noted previously, a lower SXRB flux due to a lower pressure in the hot gas does not necessarily have any impact on our calculated flux from the evaporative cloud boundary.

\subsection{LIC pressure}

The strength of the interstellar magnetic field in the LIC is unknown, though modeling of its effects on the heliosphere sets some constraints. Our best models (26 and 28) presented here have a thermal pressure of $\sim 2100 \mathrm{~cm}^{-3} \mathrm{~K}$ for the LIC. If the thermal and magnetic pressures are equal, this indicates a magnetic field strength $B \sim 2.7 \mu \mathrm{G}$, in agreement with field strengths for these models. Rough equipartition of the thermal and magnetic pressure (averaged over space and time) is found in large scale simulations of the evolution of the ISM (e.g., de Avillez $\&$ Breitschwerdt 2005), though large deviations from this are seen at any given position as a function of time. As noted is Sect. 2.3, the main effect of the field strength in the models is to regulate the pressure in the evaporative cloud boundary, which in turn affects the flux of diffuse EUV radiation incident on the cloud. The amount of EUV flux helps determine the temperature in the cloud, which is how the observational constraints fix the magnetic field strength in the context of our modeling. Thus we do not explicitly fix the magnetic field strength with the goal of achieving equipartition and indeed some of our successful models have lower or higher field strengths. It is probably coincidental that the field strength required to match the in situ $\mathrm{He}^{0}$ temperature for our best models is also close to the equipartition field strength. We note that if thermal, cosmic ray, and magnetic pressures are approximately equal the LIC has a pressure of $\sim 6300 \mathrm{~cm}^{-3} \mathrm{~K}$.

\subsection{Comparisons with other local ISM sightlines}

There have been a number of efforts to understand the local ISM ionization and abundances (Frisch et al. 1986; Cheng \& Bruhweiler 1990; Lallement \& Bertin 1992; Vallerga 1996; Lallement \& Ferlet 1997; Holberg et al. 1999; 
Kimura et al. 2003). The studies that attempt to derive gas phase elemental abundances find a range of results, generally fairly consistent with ours. A point of particular interest is the abundance of carbon that is surprisingly overabundant in our results. As an example, Kimura et al. (2003) find (based on four sightlines and excluding the $\epsilon \mathrm{CMa}$ and Sirius sightlines), a subsolar $\mathrm{C}$ abundance in contradiction with our results. Results for thirteen sightlines from Redfield \& Linsky (2004) with velocity components consistent with the LIC velocity vector show that $N(\mathrm{C}$ II $) / N(\mathrm{O}$ I $)>1$ for 8 of them, especially those at lower column density, indicating a likely solar or supersolar $\mathrm{C}$ abundance. For our best models $N(\mathrm{C}$ II $) / N(\mathrm{O} \mathrm{I}) \approx 2$. Our series of studies are unique in that we model the radiation field incident on the cloud, include radiative transfer effects, and calculate the thermal equilibrium within the cloud. The ionization varies through the cloud as does the temperature and density (slightly) and we compare observations within the heliosphere with the physical conditions at that point in the cloud rather than basing the model on line-ofsight averages.

Our present results indicate that $n(\mathrm{~N}$ II $) / n(\mathrm{~N} \mathrm{I}) \sim 0.32-0.50$ at the solar location, with $\mathrm{N}$ becoming more ionized as the sightline approaches the cloud surface. The column density ratio is thus larger, ranging from $0.38-0.62$. Observed values of $N(\mathrm{~N}$ II $) / N(\mathrm{~N}$ I $)$ toward other nearby stars are $0.58_{-0.77}^{+0.56}$ toward Capella (Wood et al. 2002), 1.29 \pm 0.23 toward HZ43 (Kruk et al. 2002), $1.91_{-0.69}^{+0.87}$ towards WD1634-573 (Lehner et al. 2003), and $1.13 \pm 0.24$ towards $\eta \mathrm{UMa}$ (Frisch et al. in preparation). The total H I column density towards each of these stars is greater than the $N(\mathrm{HI}) \sim 4 \times 10^{17} \mathrm{~cm}^{-2}$ found for the best models here. The nearest of these stars, Capella, has an ionization comparable to that of the LIC. The two high-latitude stars HZ43 and $\eta$ UMa appear to sample low opacity regions where the ionization is larger than at the Sun, as does the WD1634-573 sightline that appears to cross the nearby diffuse H II region seen towards $\lambda$ Sco (York 1983). As we have noted the $\epsilon$ CMa line of sight is special because that star is the dominant source of stellar EUV photons for the LIC. Thus for sightlines at a large angle from the $\epsilon \mathrm{CMa}$ sightline, if the H I column between points along the sightline and $\epsilon$ CMa is small the apparently high column points are subject to a strong EUV field. Such geometry dependent ionization effects can be important for non-spherical clouds subject to a strongly spatially variable ionizing radiation source.

The variation in the fractional ionization of local cloud gas has a direct impact on our understanding of the distribution and physical properties of low column density clouds for several reasons. (1) Abundances of elements with first ionization potential $<13.6 \mathrm{eV}$ must always be calculated with respect to $N(\mathrm{H} \mathrm{I})+N(\mathrm{H}$ II $)$ for very low column density clouds. (2) Cloud geometry affects the opacity of observed sightlines so that the opacity to ionizing radiation is not directly traced by the observed value of $N(\mathrm{HI})$. For lines of sight other than that towards $\epsilon \mathrm{CMa}$, this could require more complex radiative transfer models in which the difference between the line of sight toward the star and that toward one of the primary sources of ionizing flux, $\epsilon \mathrm{CMa}$, at each point is taken into account.

\section{Conclusions}

There are many uncertainties regarding the detailed properties of the ionizing interstellar radiation field incident on the local interstellar cloud (LIC). The data we have on the LIC, both from absorption line studies and in situ measurements by spacecraft in the heliosphere, provide us with strong constraints on the ionization and composition of the LIC and particularly that portion of it just outside the heliosphere, the circumheliospheric interstellar medium (CHISM). By exploring a range of models for the interstellar radiation field we find that while a fairly broad range of radiation fields can produce photoionization consistent with the data, other outputs from the models fall within a relatively narrow range of values. Our results for the models explored in this paper in which we require our models to be consistent with the LIC component of the absorption lines observed towards $\epsilon$ CMa include:

1. For a range of assumptions regarding the $\mathrm{H}$ I column density of the LIC, $N(\mathrm{HI})=(3.0-4.5) \times 10^{17} \mathrm{~cm}^{-2}$, and temperature of the hot gas of the Local Bubble, $\log T_{\mathrm{h}}=5.9,6.0$ and 6.1, we are able to find model parameters that allow a match of the model results with best observed quantities, $n\left(\mathrm{He}^{0}\right)$, $T\left(\mathrm{He}^{0}\right)$ and $N(\mathrm{Mg} \mathrm{II}) / N(\mathrm{Mg} \mathrm{I})$. For these models we assume that the cloud is evaporating because of thermal conduction between the hot Local Bubble gas and the warm LIC gas and include the emission from the cloud boundary.

2. For the best models in terms of fits to data, the required input parameters are: initial (i.e. at the outer edge of the cloud) total $\mathrm{H}$ density, $n(\mathrm{H}) \approx 0.21-0.23 \mathrm{~cm}^{-3}$; and cloud magnetic field, $B_{0} \leq 3.8 \mu \mathrm{G}$.

3. If we assume that the magnetic field configuration reduces thermal conductivity at the boundary enough to prevent evaporation and ignore any radiation from the cloud boundary, we find that for most cases the radiation field does not cause sufficient heating to maintain the LIC at the temperature observed, $T=6300 \pm 340 \mathrm{~K}$. One set of parameter choices, though, yields a successful model. These parameters are $N(\mathrm{HI})=4.5 \times 10^{17} \mathrm{~cm}^{-2}$ and $\log T_{\mathrm{h}}=6.1$.

4. Despite the wide range of possible input parameters, the output values for quantities important for shaping the heliosphere are confined to a fairly narrow range: $n\left(\mathrm{H}^{0}\right)=0.19-0.20 \mathrm{~cm}^{-3}$, and $n_{\mathrm{e}}=0.05-0.08 \mathrm{~cm}^{-3}$.

5. a $\mathrm{H}$ filtration factor of $f_{\mathrm{H}}=0.55 \pm 0.03$ yields good agreement between the radiative transfer model predictions for $n(\mathrm{HI})$ in the circumheliospheric ISM, and $n(\mathrm{HI})$ at the termination shock as found from observations of pickup ions, the H I Ly $\alpha$ glow, and the solar wind slow-down in the outer heliosphere. This filtration value is also consistent with heliosphere models of the ionization of interstellar $\mathrm{H}$ atoms traversing the heliosheath regions.

6. Elements with first ionization potentials $13.6-25 \mathrm{eV}$, e.g. $\mathrm{H}$, $\mathrm{He}, \mathrm{N}, \mathrm{O}, \mathrm{Ne}$, and $\mathrm{Ar}$, are partially ionized with ionization fractions of $\sim 0.2-0.7$.

7. By requiring that the models match the column densities derived from absorption line data we are able to determine the necessary elemental abundances for several elements. We find that the abundances of $\mathrm{N}$ and $\mathrm{O}$ may be somewhat subsolar. Sulfur is roughly solar, and C is substantially supersolar. $\mathrm{Mg}, \mathrm{Si}$ and $\mathrm{Fe}$ are all sub-solar by factors of 3-15. The depletions of $\mathrm{Fe}, \mathrm{Mg}, \mathrm{Si}$ and $\mathrm{O}$ in the LIC are consistent with a dust population consisting of amorphous silicate olivines $\mathrm{MgFeSiO}_{4}$, though other compositions for the dust are possible as well. We conclude that any carbonaceous dust in the LIC must have been destroyed, while silicate dust has persisted. Except for the gas-to-dust mass ratio, these results are in better agreement with the lower solar abundances of Grevesse et al. (2007). However we note that the $\mathrm{O}$ and $\mathrm{Ne}$ abundances of Lodders (2003) are in better agreement with other astronomical data such as the X-ray absorption edges. 
8. The gas-to-dust mass ratio derived from missing mass in the gas-phase for our best models depends strongly on the assumed reference abundance set and range from 137-323. Our two best models, Nos. 26 and 28, give a range of 149-217. For these same models $R_{\mathrm{G} / \mathrm{D}}=115-125$ based on the observed flux of dust into the heliosphere. The discrepancy of these values is minimized, in fact leading to consistency within the errors, if one assumes an abundance set such as that of GS98 which has large abundances of the metals. The GS98 abundances lead to substantial O depletion, however, which is not easily explained and conflict with the $\mathrm{S}$ abundances found for models 26 and 28.

9. These models also show that the densities of the trace ionization species $\mathrm{Ca}$ II and $\mathrm{Na}$ I are extremely sensitive to density and ionization. Therefore the ratios $N(\mathrm{Ca}$ II $) / N(\mathrm{Na} \mathrm{I})$, $N(\mathrm{Na} \mathrm{I}) / N(\mathrm{HI})$, and $N(\mathrm{Na} \mathrm{I}) / N(\mathrm{H})$ are, by themselves, inadequate diagnostics of warm low density diffuse gas.

Acknowledgements. We would like to thank George Gloeckler for sharing data with us prior to publication, and Alan Cummings for pointing out that the ACR isotopic data indicate that the LIC abundances are solar. We also thank the International Space Science Institute in Bern, Switzerland for hosting the working group on "Interstellar Hydrogen in the Heliosphere." This research was supported by NASA Solar and Heliospheric Program grants NNG05GD36G and NNG06GE33G to the University of Chicago, and by the NASA grant NNG05EC85C to SWRI.

\section{References}

Adams, T. F., \& Frisch, P. C. 1977, ApJ, 212, 300

Ajello, J. M. 1978, ApJ, 222, 1068

Altobelli, N., Krüger, H., Moissl, R., Landgraf, M., \& Grün, E. 2004, Planet Space, 52, 1287

Altun, Z., Yumak, A., Badnell, N. R., Loch, S. D., \& Pindzola, M. S. 2006, A\&A, 447, 1165

Anders, E., \& Grevesse, N. 1989, Geochim. Cosmochim. Acta, 53, 197

André, M. K., Oliveira, C. M., Howk, J. C., et al. 2003, ApJ, 591, 1000

Bellm, E. C., \& Vaillancourt, J. E. 2005, ApJ, 622, 959

Bertaux, J. L., \& Blamont, J. E. 1971, A\&A, 11, 200

Bloch, J. J., Jahoda, K., Juda, M., et al. 1986, ApJ, 308, L59

Bzowski, M. 2003, A\&A, 408, 1155

Bzowski, M., Moebius, E., Tarnopolski, S., Izmodenov, V., \& Gloeckler, G. 2008, A\&A, 491, 7

Cartledge, S. I. B., Lauroesch, J. T., Meyer, D. M., \& Sofia, U. J. 2004, ApJ, 613, 1037

Cheng, K., \& Bruhweiler, F. C. 1990, ApJ, 364, 573

Cowie, L. L., \& McKee, C. F. 1977, ApJ, 211, 135

Cravens, T. E. 2000, ApJ, 532, L153

Cummings, A. C., Stone, E. C., \& Steenberg, C. D. 2002, ApJ, 578, 194

de Avillez, M. A., \& Breitschwerdt, D. 2005, A\&A, 436, 585

Dupuis, J., Vennes, S., Bowyer, S., Pradhan, A. K., \& Thejll, P. 1995, ApJ, 455, 574

Ebel, D. S. 2000, J. Geophys. Res., 105, 10363

Egger, R. J., \& Aschenbach, B. 1995, A\&A, 294, L25

Ferland, G. J., Korista, K. T., Verner, D. A., et al. 1998, PASP, 110, 761

Field, G. B., \& Steigman, G. 1971, ApJ, 166, 59

Frisch, P., \& York, D. G. 1986, in The Galaxy and the Solar System (University of Arizona Press), 83

Frisch, P. C. 1981, Nature, 293, 377

Frisch, P. C. 2007, Composition of Matter, Space Sciences Series of ISSI (Springer), 27, 00

Frisch, P. C., \& Slavin, J. D. 2003, ApJ, 594, 844

Frisch, P. C., \& Slavin, J. D. 2005, Adv. Space Res., 35, 2048

Frisch, P. C., \& Slavin, J. D. 2006, Short Term Variations in the Galactic Environment of the Sun, in it Solar Journey: the Significance of Our Galactic Environment for the Heliosphere and Earth, ed. P. Frisch (Springer), 133

Frisch, P. C., York, D. G., \& Fowler, J. R. 1986, in ESA Special Publication, New Insights in Astrophysics, Eight Years of UV Astronomy with IUE, ed. E. J. Rolfe, 263, 491

Frisch, P. C., Dorschner, J. M., Geiss, J., et al. 1999, ApJ, 525, 492

Frisch, P. C., Grodnicki, L., \& Welty, D. E. 2002, ApJ, 574, 834

Gloeckler, G., \& Geiss, J. 2004, Adv. Space Res., 34, 53

Gloeckler, G., \& Geiss, J. 2007, Space Sci. Rev., 116
Gloeckler, G., \& Fisk, L. 2007, Composition of Matter, Space Sciences Series of ISSI (Springer), 27

Gondhalekar, P. M., Phillips, A. P., \& Wilson, R. 1980, A\&A, 85, 272

Grevesse, N., \& Sauval, A. J. 1998, Space Sci. Rev., 85, 161

Grevesse, N., Asplund, M., \& Sauval, A. J. 2007, Space Sci. Rev., 105

Gry, C., \& Jenkins, E. B. 2001, A\&A, 367, 617

Gurnett, D. A., \& Kurth, W. S. 2005, Science, 309, 2025

Hébrard, G., Mallouris, C., Ferlet, R., et al. 1999, A\&A, 350, 643

Henry, R. C. 2002, ApJ, 570, 697

Holberg, J. B., Bruhweiler, F. C., Barstow, M. A., \& Dobbie, P. D. 1999, ApJ, 517,841

Holweger, H. 2001, in Joint SOHO/ACE workshop, Solar and Galactic Composition, AIP Conf. Proc., 598, 23

Hurwitz, M., Sasseen, T. P., \& Sirk, M. M. 2004, ArXiv Astrophysics e-prints

Izmodenov, V., Malama, Y., Gloeckler, G., \& Geiss, J. 2004, A\&A, 414, L29

Juett, A. M., Schulz, N. S., Chakrabarty, D., \& Gorczyca, T. W. 2006, ApJ, 648, 1066

Kimura, H., Mann, I., \& Jessberger, E. K. 2003, ApJ, 582, 846

Koutroumpa, D., Lallement, R., Kharchenko, V., et al. 2006, A\&A, 460, 289

Kruk, J. W., Howk, J. C., André, M., et al. 2002, ApJS, 140, 19

Lallement, R., \& Bertin, P. 1992, A\&A, 266, 479

Lallement, R., \& Ferlet, R. 1997, A\&A, 324, 1105

Lallement, R., Vidal-Madjar, A., \& Ferlet, R. 1986, A\&A, 168, 225

Lallement, R., Bertin, P., Ferlet, R., Vidal-Madjar, A., \& Bertaux, J. L. 1994, A\&A, 286, 898

Lallement, R., Welsh, B. Y., Vergely, J. L., Crifo, F., \& Sfeir, D. 2003, A\&A, 411,447

Landgraf, M., Baggaley, W. J., Grün, E., Krüger, H., \& Linkert, G. 2000, J. Geophys. Res., 105, 10343

Landsman, W. B., Henry, R. C., Moos, H. W., \& Linsky, J. L. 1984, ApJ, 285, 801

Lehner, N., Jenkins, E., Gry, C., et al. 2003, ApJ, 595, 858

Leske, R. A. 2000, in 26th International Cosmic Ray Conference, ICRC XXVI, ed. B. L. Dingus, D. B. Kieda, \& M. H. Salamon, AIP Conf. Proc., 516, 274

Leske, R. A., Mewaldt, R. A., Christian, E. R., et al. 2000, in Acceleration and Transport of Energetic Particles Observed in the Heliosphere, ed. R. A. Mewaldt, J. R. Jokipii, M. A. Lee, E. Möbius, \& T. H. Zurbuchen, AIP Conf. Proc., 528, 293

Linsky, J. L., \& Wood, B. E. 1996, ApJ, 463, 254

Lodders, K. 2003, ApJ, 591, 1220

Möbius, E., Bzowski, M., Chalov, S., et al. 2004, A\&A, 426, 897

Müller, H.-R., \& Zank, G. P. 2004a, J. Geophys. Res. Space Phys., 7104

Müller, H.-R., Florinski, V., Heerikhuisen, J., et al. 2008, A\&A, 491, 43

Marschall, L. A., \& Hobbs, L. M. 1972, ApJ, 173, 43

McCammon, D., Burrows, D. N., Sanders, W. T., \& Kraushaar, W. L. 1983, ApJ, 269, 107

McCammon, D., Almy, R., Apodaca, E., et al. 2002, ApJ, 576, 188

McClintock, W., Linsky, J. L., Henry, R. C., \& Moos, H. W. 1975, ApJ, 202, 733

McClintock, W., Henry, R. C., Linsky, J. L., \& Moos, H. W. 1978, ApJ, 225, 465

Meyer, D. M., Cardelli, J. A., \& Sofia, U. J. 1997, ApJ, 490, L103

Mitchell, J. J., Cairns, I. H., \& Robinson, P. A. 2004, J. Geophys. Res., Space Physics, 109, 6108

Oliveira, C. M., Hébrard, G., Howk, J. C., et al. 2003, ApJ, 587, 235

Parravano, A., Hollenbach, D. J., \& McKee, C. F. 2003, ApJ, 584, 797

Pryor, W., Gangopadhyay, P., Sandel, W., et al. 2008, A\&A, 491, 21

Quémerais, E., Lallement, R., Bertaux, J.-L., et al. 2006a, A\&A, 455, 1135

Quémerais, E., Lallement, R., Ferron, S., et al. 2006b, J. Geophys. Res. Space Phys., 111, 9114

Raymond, J. C., \& Smith, B. W. 1977, ApJS, 35, 419

Redfield, S., \& Linsky, J. L. 2004, ApJ, 602, 776

Redfield, S., \& Linsky, J. L. 2008, ApJ, 673, 283

Richardson, J. D., Wang, C., \& Burlaga, L. F. 2004, Adv. Space Res., 34, 150

Richardson, J. D., Liu, Y., Wang, C., \& McComas, D. J. 2008, A\&A, 491, 1

Ripken, H. W., \& Fahr, H. J. 1983, A\&A, 122, 181

Robertson, I. P., \& Cravens, T. E. 2003, J. Geophys. Res., Space Phys., 108, 6

Rucinski, D., Cummings, A. C., Gloeckler, G., et al. 1996, Space Sci. Rev., 78, 73

Sanders, W. T., Edgar, R. J., Kraushaar, W. L., McCammon, D., \& Morgenthaler, J. P. 2001, ApJ, 554, 694

Savage, B. D., \& Sembach, K. R. 1996, ApJ, 470, 893

Sembach, K. R., Howk, J. C., Ryans, R. S. I., \& Keenan, F. P. 2000, ApJ, 528, 310

Simpson, J. P., Rubin, R. H., Colgan, S. W. J., Erickson, E. F., \& Haas, M. R. 2004, ApJ, 611, 338

Slavin, J. D. 1989, ApJ, 346, 718

Slavin, J. D. 2001, in XVII IAP Colloquium on Gaseous Matter in Galaxies and Intergalactic Space, ed. J.-M. D. R. Ferlet, \& M. Lemoine (Frontier Group), 27 
Slavin, J. D., \& Frisch, P. C. 2002, ApJ, 565, 364

Slavin, J. D., \& Frisch, P. C. 2006, ApJ, 651, L37

Smith, R. K., Edgar, R. J., Plucinsky, P. P., et al. 2005, ApJ, 623, 225

Snowden, S. L., Cox, D. P., McCammon, D., \& Sanders, W. T. 1990, ApJ, 354 211

Snowden, S. L., McCammon, D., \& Verter, F. 1993, ApJ, 409, L21

Snowden, S. L., Egger, R., Freyberg, M. J., et al. 1997, ApJ, 485, 125

Snowden, S. L., Egger, R., Finkbeiner, D. P., Freyberg, M. J., \& Plucinsky, P. P. 1998, ApJ, 493, 715

Snowden, S. L., Collier, M. R., \& Kuntz, K. D. 2004, ApJ, 610, 1182

Sofia, U. J., Cardelli, J. A., Guerin, K. P., \& Meyer, D. M. 1997, ApJ, 482, L105 Stokes, G. M. 1978, ApJS, 36, 115

Takei, Y., Fujimoto, R., Mitsuda, K., \& Onaka, T. 2002, ApJ, 581, 307

Thomas, G. E., \& Krassa, R. F. 1971, A\&A, 11, 218
Vallerga, J. 1996, Space Sci. Rev., 78, 277

Vallerga, J. 1998, ApJ, 497, 921

Wargelin, B. J., Markevitch, M., Juda, M., et al. 2004, ApJ, 607, 596

Weller, C. S., \& Meier, R. R. 1981, ApJ, 246, 386

Welty, D. E., Hobbs, L. M., Lauroesch, J. T., et al. 1999, ApJS, 124, 465

Witte, M. 2004, A\&A, 426, 835

Witte, M., Banaszkiewicz, M., \& Rosenbauer, H. 1996, Space Sci. Rev., 78, 289

Wood, B. E., Linsky, J. L., \& Zank, G. P. 2000a, ApJ, 537, 304

Wood, B. E., Linsky, J. L., \& Zank, G. P. 2000b, ApJ, 537, 304

Wood, B. E., Redfield, S., Linsky, J. L., \& Sahu, M. S. 2002, ApJ, 581, 1168

Wood, B. E., Redfield, S., Linsky, J. L., Müller, H.-R., \& Zank, G. P. 2005, ApJS,

159,118

York, D. G. 1974, ApJ, 193, L127

York, D. G. 1983, ApJ, 264, 172 